\title{
VOLUME CONJECTURES FOR THE RESHETIKHIN-TURAEV AND THE TURAEV-VIRO INVARIANTS
}

\author{
QINGTAO CHEN AND TIAN YANG
}

\begin{abstract}
We consider the asymptotics of the Turaev-Viro and the Reshetikhin-Turaev invariants of a hyperbolic 3-manifold, evaluated at the root of unity $\exp (2 \pi \sqrt{-1} / r)$ instead of the standard $\exp (\pi \sqrt{-1} / r)$. We present evidence that, as $r$ tends to $\infty$, these invariants grow exponentially with growth rates respectively given by the hyperbolic and the complex volume of the manifold. This reveals an asymptotic behavior that is different from that of Witten's Asymptotic Expansion Conjecture, which predicts polynomial growth of these invariants when evaluated at the standard root of unity. This new phenomenon suggests that the Reshetikhin-Turaev invariants may have a geometric interpretation other than the original one via $S U(2)$ Chern-Simons gauge theory.
\end{abstract}

\section{INTRODUCTION}

In [62], Witten provided a new interpretation of the Jones polynomial based on Chern-Simons gauge theory, and expanded on this idea to construct a sequence of complex valued 3-manifold invariants. This approach was formalized though the representation theory of quantum groups by Reshetikhin and Turaev [53, 54], who generalized the Jones polynomial to a sequence of polynomial invariants of a link, later called the colored Jones polynomials of that link. They also defined a sequence of 3-manifold invariants corresponding to Witten's invariants. The Reshetikhin-Turaev construction of 3-manifold invariants starts from a surgery description [33] of the manifold, and evaluates the colored Jones polynomials of the surgery data at certain roots of unity.

A different approach was developed by Turaev and Viro [60] who, from a triangulation of a closed 3-manifold, constructed real valued invariants of the manifold by using quantum $6 j$ symbols [35]; these Turaev-Viro invariants turned out to be equal to the square of the norm of the Reshetikhin-Turaev invariants [55, 59, 61].

Using quantum dilogarithm functions, Kashaev [29, 30] used a different type of $6 j$-symbols, involving the discrete quantum dilogarithm, to define for each integer $n$ complex valued link invariants. He observed in a few examples, and conjectured in the general case, that the absolute value of these invariants grow exponentially with $n$, and that the growth rate is given by the hyperbolic volume of the complement of the link. In [44], Murakami and Murakami showed that Kashaev's invariants coincide with the values of the colored Jones polynomials at a certain root of unity, and reformulated Kashaev's conjecture as follows.

Volume Conjecture $\left([[30,44])\right.$. For a hyperbolic link $L$ in $S^{3}$, let $J_{n}(L ; q)$ be its $n$-th colored Jones polynomial. Then

$$
\lim _{n \rightarrow+\infty} \frac{2 \pi}{n} \log \left|J_{n}\left(L ; e^{\frac{2 \pi \sqrt{-1}}{n}}\right)\right|=\operatorname{vol}\left(S^{3} \backslash L\right),
$$

where $\operatorname{vol}\left(S^{3} \backslash L\right)$ is the hyperbolic volume of the complement of $L$.

The research of the first author was partially supported by the National Centre of Competence in Research SwissMAP of the Swiss National Science Foundation. The second author was supported by NSF grant DMS-1405066. 
This conjecture has now been proved for a certain number of cases: the figure-eight knot [44]; all hyperbolic knots with at most six crossings [49, 50]; the Borromean rings [24]; the twisted Whitehead links [66]; the Whitehead chains [63]. Various extensions of this conjecture have been proposed, and proved for certain cases in [28, 46, 15, 20, 19, 47].

In the current paper we investigate the asymptotic behavior of the Reshetikhin-Turaev and the Turaev-Viro invariants, evaluated at the root of unity $q=e^{\frac{2 \pi \sqrt{-1}}{r}}$. Supported by numerical evidence, we propose the following conjecture.

Conjecture 1.1. For a hyperbolic 3-manifold $M$, let $\mathrm{TV}_{r}(M ; q)$ be its Turaev-Viro invariant and let $\operatorname{vol}(M)$ be its hyperbolic volume. Then for $r$ running over all odd integers and for $q=e^{\frac{2 \pi \sqrt{-1}}{r}}$,

$$
\lim _{r \rightarrow+\infty} \frac{2 \pi}{r} \log \left(\mathrm{TV}_{r}(M ; q)\right)=\operatorname{vol}(M) .
$$

We here consider all types of hyperbolic 3-manifolds: closed, cusped or those with totally geodesic boundary. The Turaev-Viro invariant $\operatorname{TV}_{r}(M ; q)$ is the original one defined in [60] when the manifold $M$ is closed, and is its extension defined in [10] when $M$ has non-empty boundary. See $\$ 2.3$ for details.

This conjecture should be contrasted with Witten's Asymptotic Expansion Conjecture (see [48]) which predicts that, when evaluated at $q=e^{\frac{\pi \sqrt{-1}}{r}}$, the Witten invariants of a 3-manifold (and therefore its Reshetikhin-Turaev and Turaev-Viro invariants) only grow polynomially, with a growth rate related to classical invariants of the manifold such as the Chern-Simons invariant and the Reidemeister torsion.

Conjecture 1.1 is motivated by the beautiful work of Costantino [16] relating the asymptotics of quantum $6 j$-symbols to the volumes of truncated hyperideal tetrahedra. See also [18, 19].

We provide much supporting evidence for Conjecture 1.1 . In $\$ 3$, we numerically calculate $\mathrm{TV}_{r}(M)$ for various hyperbolic 3-manifolds with cusps, including the figure-eight knot complement and its sister, the complements of the knots $K_{5_{2}}$ and $K_{6_{1}}$, and the manifolds denoted by $M_{3_{6}}$, $M_{38}, N_{1_{1}}$ and $N_{2_{1}}$ in the Callahan-Hildebrand-Weeks census [14]. We also numerically calculate $\mathrm{TV}_{r}(M)$ for the smallest hyperbolic 3-manifolds with a totally geodesic boundary [23, 39].

Recently, Detcherry, Kalfagianni and the second author [22] provided a rigorous proof of Conjecture 1.1 for the figure-eight knot complement.

The Reshetikhin-Turaev invariants $\mathrm{RT}_{r}(M ; q)$ are complex valued invariants of a closed oriented 3-manifold $M$, defined for all integers $r \geqslant 3$ and all primitive $2 r$-th roots of unity $q$. For $q=e^{\frac{\pi \sqrt{-1}}{r}}$, these invariants provide a mathematical realization of Witten's invariants [62]. Following a skein theory approach pioneered by Lickorish [36, 37], Blanchet-Habegger-Masbaum-Vogel [11] (see also Lickorish [38]) extended Reshetikhin-Turaev invariants to primitive $r$-th roots of unity $q$ with $r$ odd. In particular, $\operatorname{RT}_{r}(M ; q)$ is defined at $q=e^{\frac{2 \pi \sqrt{-1}}{r}}$ when $r$ is odd. In $\$ 4$. we numerically compute Reshetikhin-Turaev invariants for various closed hyperbolic 3-manifolds obtained by integral Dehn surgery along the knots $K_{4_{1}}$ and $K_{5_{2}}$. These calculations suggest the following conjecture.

Conjecture 1.2. Let $M$ be a closed oriented hyperbolic 3-manifold and let $\operatorname{RT}_{r}(M ; q)$ be its Reshetikhin-Turaev invariants. Then for $q=e^{\frac{2 \pi \sqrt{-1}}{r}}$ with $r$ odd and for a suitable choice of the arguments,

$$
\lim _{r \rightarrow+\infty} \frac{4 \pi \sqrt{-1}}{r} \log \left(\operatorname{RT}_{r}(M ; q)\right)=\operatorname{CS}(M)+\operatorname{vol}(M) \sqrt{-1} \bmod \pi^{2} \mathbb{Z}
$$


where $\operatorname{CS}(M)$ denotes the Chern-Simons invariant of the hyperbolic metric of $M$ multiplied by $2 \pi^{2}$.

Ohtsuki [51] recently announced a proof of Conjecture 1.2 for the manifolds obtained by Dehn surgery along the knot $K_{4_{1}}$. By [55, 59, 61], Conjecture [1.2 implies Conjecture 1.1 for closed 3-manifolds.

Comparing Conjecture 1.2 with Witten's Asymptotic Expansion Conjecture, one sees a very different asymptotic behavior for the Reshetikhin-Turaev invariants evaluated at $q=e^{\frac{2 \pi \sqrt{-1}}{r}}$ and $q=e^{\frac{\pi \sqrt{-1}}{2 r}}$. Our numerical calculations also suggest exponential growth at other roots of unity such as $q=e^{\frac{3 \pi \sqrt{-1}}{r}}$. For these roots of unity, we expect a geometric interpretation of Reshetikhin-Turaev invariants that is different from the $S U(2)$ Chern-Simons gauge theory.

In $\$ 5$, we calculate $\mathrm{TV}_{r}(M)$ for the complements of the unknot, the Hopf link, the trefoil knot and the torus links $T_{(2,4)}$ and $T_{(2,6)}$. We also numerically calculate $\mathrm{TV}_{r}(M)$ for the complement of the torus knots $T_{(2,5)}, T_{(2,7)}, T_{(2,9)}, T_{(2,11)}, T_{(3,5)}$ and $T_{(3,7)}$. These computations suggest an Integrality Conjecture (Conjecture 5.1) which states that the Turaev-Viro invariants of torus link complement are integers independent of the roots of unity at which they are evaluated.

Acknowledgments: Part of this work was done during the Tenth East Asian School of Knots and Related Topics at the East China Normal University in January 2015. We would like to thank the organizers for their support and hospitality.

The authors are deeply grateful to Francis Bonahon for discussions, suggestions and improving the writing of the paper, and are also grateful to Riccardo Benedetti, Francesco Costantino, Charles Frohman, Stavros Garoufalidis, Rinat Kashaev, Liang Kong, Thang Lê, Julien Marché, Gregor Masbaum, Nicolai Reshetikhin, Dylan Thurston, Roland van der Veen, Zhenghan Wang and Hao Zheng for discussions and suggestions, and Xiaogang Wen and Shing-Tung Yau for showing interest in this work. The first author thanks Kefeng Liu and Weiping Zhang for many useful discussions during the past few years, and Nicolai Reshetikhin for guiding him to the area of quantum invariants and sharing many experiences and ideas since 2005. The second author thanks Henry Segerman and Hongbin Sun for very helpful discussions, and Steven Kerckhoff, Feng Luo and Maryam Mirzakhani for many suggestions.

\section{PRELIMINARIES}

We recall the construction of Turaev-Viro invariants of 3-dimensional manifolds. In order to follow a uniform treatment for all cases, we extend their definition to pseudo 3-manifolds.

2.1. Pseudo 3-manifolds and triangulations. A pseudo 3-manifold is a topological space $M$ such that each point $p$ of $M$ has a neighborhood $U_{p}$ that is homeomorphic to a cone over a surface $\Sigma_{p}$. We call $p$ a singular point and $U_{p}$ a singular neighborhood if $\Sigma_{p}$ is not a 2 -sphere. In particular, a closed 3-manifold is a pseudo 3-manifold with no singular point, and every 3-manifold with boundary is homeomorpic to a pseudo 3-manifold with suitable singular neighborhoods of all singular points removed.

A triangulation $\mathcal{T}$ of a pseudo manifold $M$ consists of a disjoint union $X=\bigsqcup \Delta_{i}$ of finitely many Euclidean tetrahedra $\Delta_{i}$ and of a collection of homeomorphisms $\Phi$ between pairs of faces in $X$ such that the quotient space $X / \Phi$ is homeomorphic to $M$. The vertices, edges, faces and tetrahedra in $\mathcal{T}$ are respectively the quotients of the vertices, edges, faces and tetrahedra in $X$. From the definition, we see that a singular point of $M$ must be a vertex of $\mathcal{T}$. We call the nonsingular vertices of $\mathcal{T}$ the inner vertices. If $M$ is a closed 3-manifold, then a triangulation of $M$ is 
a triangulation of manifold in the usual sense; and if $N$ is a 3-manifold with boundary obtained by removing all singular neighborhoods of a pseudo 3-manifold $M$, then a triangulation of $M$ without inner vertices determines an ideal triangulation of $N$.

In [41, 42, 52], it is proved that any two triangulations of a pseudo 3-manifold are related by a sequence of 0-2 and 2-3 Pachner moves. See the figure below, where in the $0-2$ move a new inner vertex is introduced.

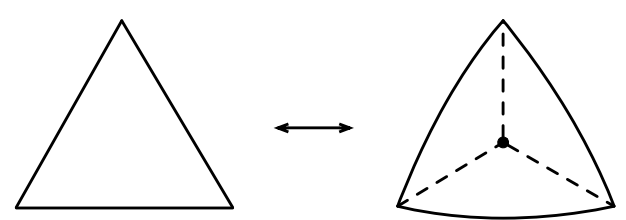

0-2 move

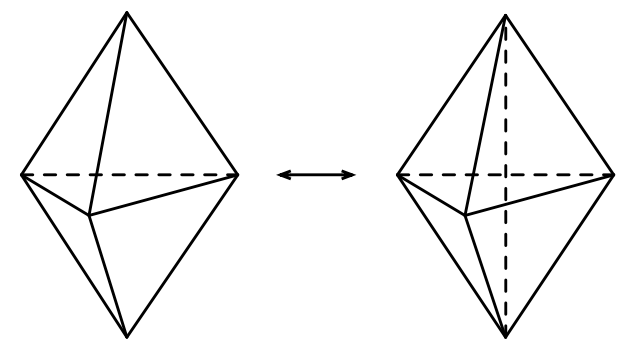

2-3 move

2.2. Quantum 6j-symbols. We now recall the definition and basic properties of the quantum $6 j$-symbols. See [35, 32] for more details.

Throughout this subsection, we will fix an integer $r \geqslant 3$, and we let $I_{r}=\{0,1 / 2, \ldots,(r-2) / 2\}$ be the set of non-negative half-integers less than or equal to $(r-2) / 2$. The elements of $I_{r}$ are traditionally called colors.

Let $q \in \mathbb{C}$ be a root of unity such that $q^{2}$ is a primitive root of unity of order $r$. For an integer $n$, the quantum integer $[n]$ is the real number defined by

$$
[n]=\frac{q^{n}-q^{-n}}{q-q^{-1}}
$$

and the associated quantum factorial is $[n] !=[n][n-1] \ldots[1]$. By convention, $[0] !=1$.

A triple $(i, j, k)$ of elements of $I_{r}$ is called admissible if

(1) $i+j \geqslant k, j+k \geqslant i$ and $k+i \geqslant j$,

(2) $i+j+k \in \mathbb{Z}$,

(3) $i+j+k \leqslant r-2$.

A 6-tuple $(i, j, k, l, m, n)$ of elements of $I_{r}$ is admissible if the triples $(i, j, k),(j, l, n),(i, m, n)$ and $(k, l, m)$ are admissible

For an admissible triple $(i, j, k)$, define

$$
\Delta(i, j, k)=\sqrt{\frac{[i+j-k] ![j+k-i] ![k+i-j] !}{[i+j+k+1] !}}
$$

with the convention that $\sqrt{x}=\sqrt{|x|} \sqrt{-1}$ when the real number $x$ is negative. 
Definition 2.1. The quantum $6 j$-symbol of an admissible 6-tuple $(i, j, k, l, m, n)$ is the number

$$
\begin{aligned}
\left|\begin{array}{ccc}
i & j & k \\
l & m & n
\end{array}\right|= & \sqrt{-1}^{-2(i+j+k+l+m+n)} \Delta(i, j, k) \Delta(j, l, n) \Delta(i, m, n) \Delta(k, l, m) \\
& \sum_{z=\min \left\{Q_{1}, Q_{2}, Q_{3}\right\}} \frac{(-1)^{z}[z+1] !}{\left[z-T_{1}, T_{2}, T_{3}, T_{4}\right\} !\left[z-T_{2}\right] !\left[z-T_{3}\right] !\left[z-T_{4}\right] !\left[Q_{1}-z\right] !\left[Q_{2}-z\right] !\left[Q_{3}-z\right] !}
\end{aligned}
$$

where $T_{1}=i+j+k, T_{2}=j+l+n, T_{3}=i+m+n$ and $T_{4}=k+l+m, Q_{1}=i+j+l+m$, $Q_{2}=i+k+l+n$ and $Q_{3}=j+k+m+n$.

A good way to memorize the definitions is to consider a tetrahedron as in Figure 1, and to attach the weights $i, j, k, l, m, n$ to its edges as indicated in the figure. Then each of $T_{1}, T_{2}, T_{3}, T_{4}$ corresponds to a face of the tetrahedron, and each of $Q_{1}, Q_{2}, Q_{3}$ corresponds to a quadrilateral separating two pairs of the vertices.

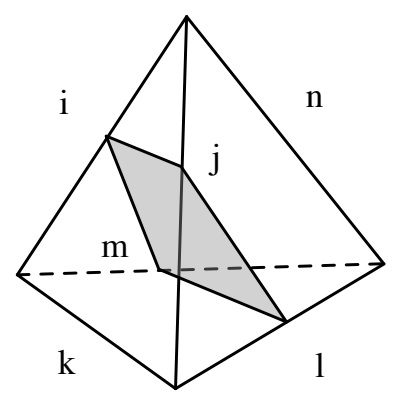

FIGURE $1.6 j$-symbols and the tetrahedron

The following symmetries

$$
\left|\begin{array}{ccc}
i & j & k \\
l & m & n
\end{array}\right|=\left|\begin{array}{ccc}
j & i & k \\
m & l & n
\end{array}\right|=\left|\begin{array}{ccc}
i & k & j \\
l & n & m
\end{array}\right|=\left|\begin{array}{ccc}
i & m & n \\
l & j & k
\end{array}\right|=\left|\begin{array}{ccc}
l & m & k \\
i & j & n
\end{array}\right|=\left|\begin{array}{ccc}
l & j & n \\
i & m & k
\end{array}\right|
$$

immediately follow from the definitions.

The quantum $6 j$-symbols satisfy the following two important identities, which are crucial in the construction of the Turaev-Viro invariants. For $i \in I_{r}$, set

$$
w_{i}=(-1)^{2 i}[2 i+1] \quad \text { and } \quad \eta=\sum_{i \in I_{r}} w_{i}^{2} .
$$

Proposition 2.2 (Orthogonality Property). For any admissible 6-tuple (i,j, $k, l, m, n$ ),

$$
\sum_{s} w_{s} w_{m}\left|\begin{array}{ccc}
i & j & m \\
k & l & s
\end{array}\right|\left|\begin{array}{ccc}
i & j & n \\
k & l & s
\end{array}\right|=\delta_{m n}
$$

where $\delta$ is the Kronecker symbol, and where the sum is over all $s \in I_{r}$ such that the two 6-tuples in the sum are admissible.

Corollary 2.3. For any admissible triple $(i, j, k)$,

$$
\eta^{-1} \sum_{l, m, n} w_{l} w_{m} w_{n}\left|\begin{array}{ccc}
i & j & k \\
l & m & n
\end{array}\right|\left|\begin{array}{ccc}
i & j & k \\
l & m & n
\end{array}\right|=1
$$


where the sum is over $l, m, n \in I_{r}$ such that the 6-tuples $(i, j, k, l, m, n)$ is admissible.

Proposition 2.4 (Biedenharn-Elliot identity). For any $i, j, k, l, m, n, o, p, q \in I_{r}$ such that $(o, p, q, i, j, k)$ and $(o, p, q, l, m, n)$ are admissible,

$$
\sum_{s} w_{s}\left|\begin{array}{ccc}
i & j & q \\
m & l & s
\end{array}\right|\left|\begin{array}{ccc}
j & k & o \\
n & m & s
\end{array}\right|\left|\begin{array}{ccc}
k & i & p \\
l & n & s
\end{array}\right|=\left|\begin{array}{ccc}
o & p & q \\
i & j & k
\end{array}\right|\left|\begin{array}{ccc}
o & p & q \\
l & m & n
\end{array}\right|,
$$

where the sum is over $s \in I_{r}$ such that the three 6-tuples in the sum are admissible.

2.3. Turaev-Viro invariants of pseudo 3-manifolds. Let $q$ be a root of unity, and let $r$ be such that $q^{2}$ is a primitive root of unity of order $r$. As in $\$ 2.2$, we consider the set $I_{r}=\{0,1 / 2,1, \ldots,(r-$ 2) $/ 2\}$ of colors, and the notation

$$
[n]=\frac{q^{n}-q^{-n}}{q-q^{-1}}, \quad w_{i}=(-1)^{2 i}[2 i+1], \quad \eta=\sum_{i \in I_{r}} w_{i}^{2} .
$$

for every integer $n$ and color $i \in I_{r}$.

For a triangulation $\mathcal{T}$ of a pseudo 3-manifold $M$, an $r$-admissible coloring of $(M, \mathcal{T})$ is a map

$$
c:\{\text { edges of } \mathcal{T}\} \rightarrow I_{r}
$$

such that, for every 2-dimensional face $F$ of $\mathcal{T}$, the colors $c\left(e_{1}\right), c\left(e_{2}\right), c\left(e_{3}\right) \in \mathcal{T}$ associated to the edges of $F$ form an admissible triple. Such a coloring $c$ associates to each edge $e$ of $\mathcal{T}$ the number

$$
|e|_{c}=w_{c(e)}
$$

and to each tetrahedron $\Delta$ of $\mathcal{T}$ the $6 j$-symbol

$$
|\Delta|_{c}=\left|\begin{array}{lll}
c\left(e_{12}\right) & c\left(e_{13}\right) & c\left(e_{23}\right) \\
c\left(e_{34}\right) & c\left(e_{24}\right) & c\left(e_{14}\right)
\end{array}\right|
$$

where the edges of $\Delta$ are indexed in such a way that, if $v_{1}, v_{2}, v_{3}, v_{4}$ denote the vertices of $\Delta$, the edge $e_{i j}$ connects $v_{i}$ to $v_{j}$.

Definition 2.5. With the above definitions, the Turaev-Viro invariant of $M$ associated to the root of unity $q$ is defined as the sum

$$
\operatorname{TV}_{q}(M, \mathcal{T})=\eta^{-|V|} \sum_{c \in A_{r}} \prod_{e \in E}|e|_{c} \prod_{\Delta \in T}|\Delta|_{c}
$$

where $V, E, T, A_{r}$ respectively denote the sets of inner vertices, edges, tetrahedra and $r$-admissible colorings of the triangulation $\mathcal{T}$.

Theorem 2.6. The above invariant $\operatorname{TV}_{q}(M, \mathcal{T})$ depends only on the pseudo-manifold $M$ and on the root of unity $q$, not on the triangulation $\mathcal{T}$.

Proof. Theorem 2.6 is proved by a straightforward extension to pseudo 3-manifolds of the original argument of Turaev and Viro in [60] for 3-manifolds.

The first ingredient is a purely topological statement, proved in [41, 42, 52], which says that any two triangulations of a pseudo 3-manifold are related by a sequence of the Pachner Moves 0-2 and 2-3 represented in Figures 2 and 3. The Pachner Move 0-2 replaces a 2-dimensional face of the triangulation by two tetrahedra meeting along 3 faces, and adds one vertex to the triangulation. The 2-3 Move replaces two tetrahedra meeting along one face by three tetrahedra sharing one edge.

The second ingredient is algebraic, and is provided by the properties of $6 j$-symbols given in $\$ 2.2$. Indeed, exactly as in [60], Corollary 2.3 of the Orthogonality Property of Proposition 2.1 


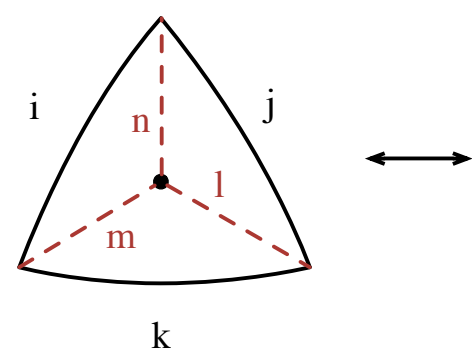

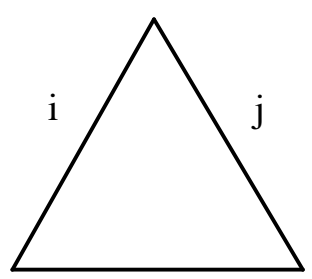

$\mathrm{k}$

FIgURE 2. The Pachner Move 0-2
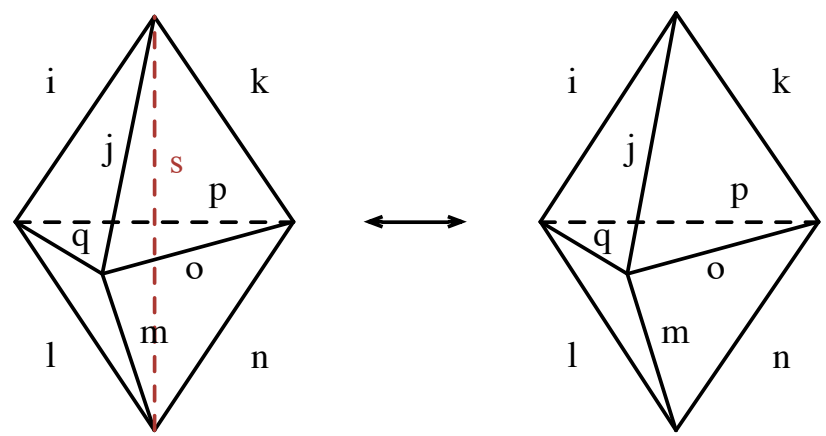

FiguRE 3. The Pachner Mover 2-3

shows that $\operatorname{TV}_{r}(M, \mathcal{T})$ is unchanged as we modify the triangulation $\mathcal{T}$ by a $0-2$ move, and the Biedenharn-Elliot identity (2.3) guarantees the invariance under the 2-3 move.

As mentioned in $\$ 2.1$, a triangulation of a pseudo 3-manifold without inner vertices determines an ideal triangulation of the 3-manifold with boundary obtained by removing all the singular neighborhoods. Hence for a 3-manifold $M$ with boundary, one can define $\mathrm{TV}_{r}(M)$ using an ideal triangulation of $M$. Our invariant (and its construction) then coincides with the one defined in [10] using o-graphs.

Theorem 2.6 shows that, for any $r$ and $q$ as above, $\operatorname{TV}_{q}(M, \mathcal{T})$ is independent of the choice of of the triangulation $\mathcal{T}$. We will consequently omit the triangulation $\mathcal{T}$ and denote the invariant by $\mathrm{TV}_{r}(M)$ if $q=e^{\frac{2 \pi \sqrt{-1}}{r}}$, or by $\operatorname{TV}_{r}(M ; q)$ if we want to emphasize which root of unity $q$ is being used.

\section{EVIDENCE FOR 3-MANIFOLDS WITH BOUNDARY}

We now provide numerical evidence for Conjecture 1.1 for a few hyperbolic 3-manifolds with boundary. The closed manifold case will be considered in the next section. The reason for considering the two cases separately is that a hyperbolic 3-manifold with boundary often admits an ideal triangulation by a small number of tetrahedra, whereas a triangulation of a closed hyperbolic 3-manifold usually requires more tetrahedra. For example, it takes at least nine tetrahedra to triangulate the Weeks manifold, which is the smallest closed hyperbolic 3-manifold.

To simplify the notation, set

$$
Q V_{r}(M)=\frac{2 \pi}{r-2} \log \left(\mathrm{TV}_{r}\left(M ; e^{\frac{2 \pi \sqrt{-1}}{r}}\right)\right)
$$


for each odd integer $r \geqslant 3$. Similarly, write

$$
\mathrm{TV}_{r}(L)=\mathrm{TV}_{r}\left(S^{3} \backslash L\right) \text { and } \quad Q V_{r}(L)=Q V_{r}\left(S^{3} \backslash L\right)
$$

when $M=S^{3} \backslash L$ is a link complement.

3.1. The figure-eight knot complement and its sister. By Thurston's famous construction [56], the figure-eight knot complement $S^{3} \backslash K_{4_{1}}$ has volume

$$
\operatorname{vol}\left(S^{3} \backslash K_{4_{1}}\right) \approx 2.02988,
$$

and has the ideal triangulation represented in Figure 4. In that figure, edges with the same labels $(a$ or $b)$ are glued together following the indicated orientations to form an edge of the ideal triangulation.
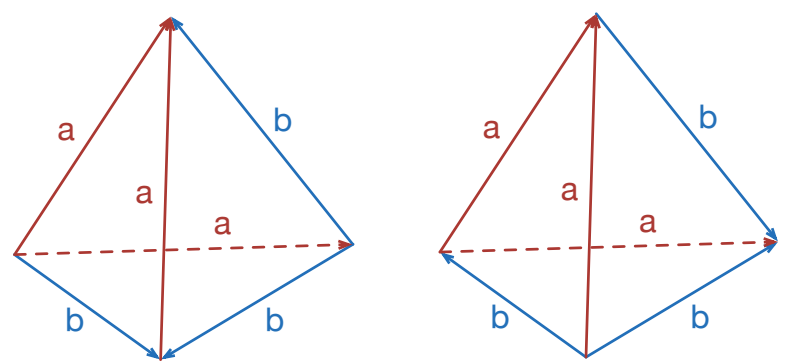

FIGURE 4.

By Definition 2.5, we have

$$
\mathrm{TV}_{r}\left(K_{4_{1}}\right)=\sum_{(a, b) \in A_{r}} w_{a} w_{b}\left|\begin{array}{lll}
a & a & b \\
a & b & b
\end{array}\right|\left|\begin{array}{lll}
a & a & b \\
a & b & b
\end{array}\right|,
$$

where $A_{r}$ consists of the pairs $(a, b)$ of elements of $I_{r}$ such that $(a, a, b)$ and $(b, b, a)$ are admissible, i.e., $2 a-b \geqslant 0,2 b-a \geqslant 0,2 a+b \leqslant r-2,2 b+a \leqslant r-2$ and $2 a+b$ and $2 b+a$ are integers. From this formula, we have the following table of values of $Q V_{r}\left(K_{4_{1}}\right)$.

\begin{tabular}{|c||c|c|c|c|c|c|c|c|c|}
\hline $\mathrm{r}$ & 11 & 13 & 15 & 17 & 19 & 21 & 23 & 25 & 31 \\
\hline$Q V_{r}\left(K_{4_{1}}\right)$ & 2.40661 & 2.37755 & 2.34826 & 2.31907 & 2.29953 & 2.28227 & 2.26834 & 2.25634 & 2.22824 \\
\hline \hline$r$ & 41 & 51 & 61 & 71 & 81 & 91 & 101 & 111 & 121 \\
\hline$Q V_{r}\left(K_{4_{1}}\right)$ & 2.19685 & 2.17540 & 2.15953 & 2.14721 & 2.13731 & 2.12915 & 2.12230 & 2.11643 & 2.11136 \\
\hline$r$ & 131 & 141 & 151 & 201 & 301 & 401 & 501 & 701 & 1001 \\
\hline$Q V_{r}\left(K_{4_{1}}\right)$ & 2.10692 & 2.10299 & 2.09949 & 2.08641 & 2.07168 & 2.06344 & 2.05810 & 2.05153 & 2.04614 \\
\hline
\end{tabular}

Figure 5 below compares the values of the Turaev-Viro invariants $Q V_{r}\left(K_{4_{1}}\right)$ and the Kashaev invariants $\left\langle K_{4_{1}}\right\rangle_{r}$ for various values of $r$. The dots represent the points $\left(r, Q V_{r}\left(K_{4_{1}}\right)\right)$, the diamonds represent the points $\left(r, \frac{2 \pi}{r} \log \left|\left\langle K_{4_{1}}\right\rangle_{r}\right|\right)$, and the squares represent the points $\left(r, \operatorname{vol}\left(S^{3} \backslash K_{4_{1}}\right)\right)$. Note that the values of $Q V_{r}\left(K_{4_{1}}\right)$ appear to converge to $\operatorname{vol}\left(S^{3} \backslash K_{4_{1}}\right)$ much faster than $\left\langle K_{4_{1}}\right\rangle_{r}$ as $r$ becomes large.

The manifold $M_{2_{2}}$ in the Callahan-Hildebrand-Weeks census [14], also known as the figure-eight sister, shares the same volume with the figure-eight knot complement, i.e.,

$$
\operatorname{vol}\left(M_{2_{2}}\right)=\operatorname{vol}\left(S^{3} \backslash K_{4_{1}}\right) \approx 2.02988 .
$$




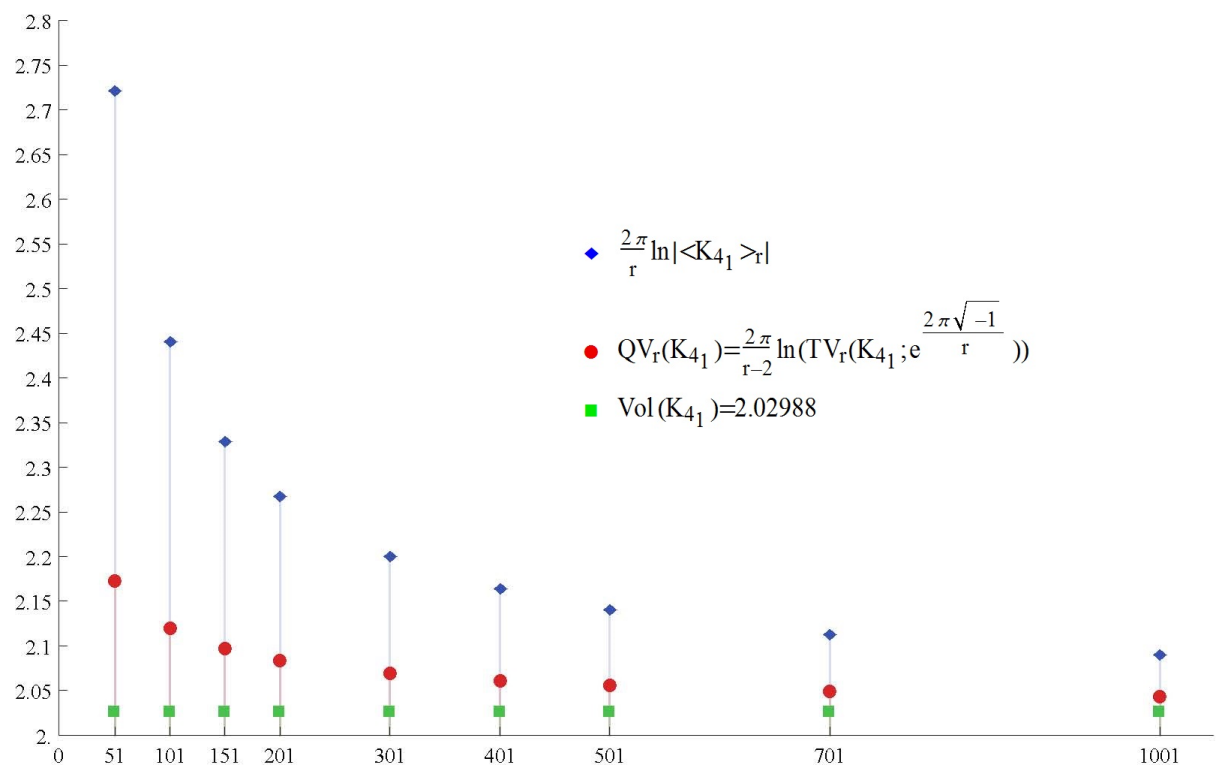

FIGURE 5. Comparison of different invariants for $K_{4_{1}}$

It is also known that $M_{2_{2}}$ is not the complement of any knot in $S^{3}$. According to Regina [12], $M_{2_{2}}$ has the ideal triangulation represented in Figure 6.
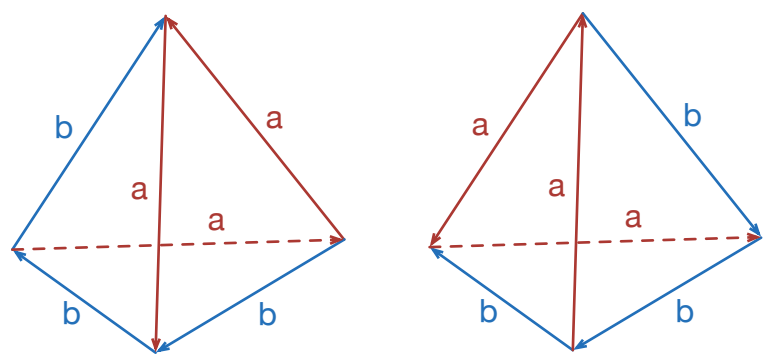

FIGURE 6.

Since for each tetrahedron in this triangulation, the coloring is the same as that of $S^{3} \backslash K_{4_{1}}$, the invariant $\mathrm{TV}_{r}\left(M_{2_{2}}\right)$ has exactly the same formula as $\operatorname{TV}_{r}\left(K_{4_{1}}\right)$. As a consequence, the TuraevViro invariants of these manifolds take the same values.

3.2. The $K_{5_{2}}$ knot complement and its sisters. According to SnapPy [21] and Regina [12], the complement of the knot $K_{5_{2}}$ has volume

$$
\operatorname{vol}\left(S^{3} \backslash K_{5_{2}}\right) \approx 2.82812,
$$

and admits the ideal triangulation represented in Figure 7. Since only the colors of the edges (according to which the edges are identified to form an edge of the triangulation) matters in the calculation of $\mathrm{TV}_{r}(M)$, we omit the arrows on the edges.

By Definition 2.5, we have

$$
\operatorname{TV}_{r}\left(K_{5_{2}}\right)=\sum_{(a, b, c) \in A_{r}} w_{a} w_{b} w_{c}\left|\begin{array}{lll}
a & a & b \\
b & c & c
\end{array}\right|\left|\begin{array}{lll}
a & a & b \\
b & c & c
\end{array}\right|\left|\begin{array}{lll}
a & b & c \\
b & b & c
\end{array}\right|,
$$



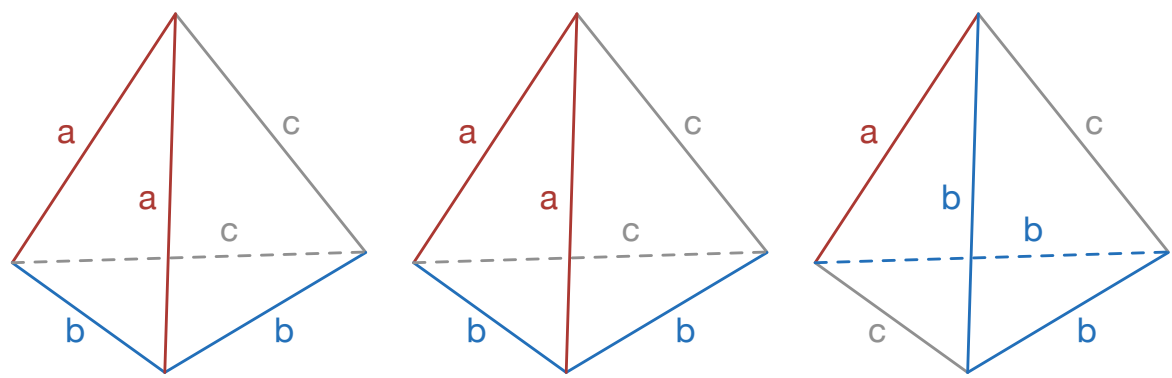

FIGURE 7.

where $A_{r}$ consists of triples $(a, b, c)$ of elements of $I_{r}$ such that $(a, a, b),(b, b, c),(c, c, a)$ and $(a, b, c)$ are admissible. From this, we have the following table of values of $Q V_{r}\left(K_{5_{2}}\right)$.

\begin{tabular}{|c||c|c|c|c|c|c|c|c|}
\hline $\mathrm{r}$ & 7 & 9 & 11 & 21 & 31 & 41 & 51 & 61 \\
\hline$Q V_{r}\left(K_{5_{2}}\right)$ & 3.38531 & 3.32394 & 3.25282 & 3.09588 & 3.03657 & 3.00236 & 2.97925 & 2.96232 \\
\hline$r$ & 71 & 81 & 91 & 101 & 121 & 151 & 201 & 301 \\
\hline$Q V_{r}\left(K_{5_{2}}\right)$ & 2.94927 & 2.93883 & 2.93027 & 2.92309 & 2.91169 & 2.89937 & 2.88586 & 2.87071 \\
\hline
\end{tabular}

Figure 8 compares the values of $Q V_{r}\left(K_{5_{2}}\right)$ with those of the Kashaev invariants $\left\langle K_{5_{2}}\right\rangle_{r}$. Again, the dots represent the points $\left(r, Q V_{r}\left(K_{5_{2}}\right)\right)$, the diamonds represent the points $\left(r, \frac{2 \pi}{r} \log \left|\left\langle K_{5_{2}}\right\rangle_{r}\right|\right)$, and the squares represent the points $\left(r, \operatorname{vol}\left(S^{3} \backslash K_{5_{2}}\right)\right)$.

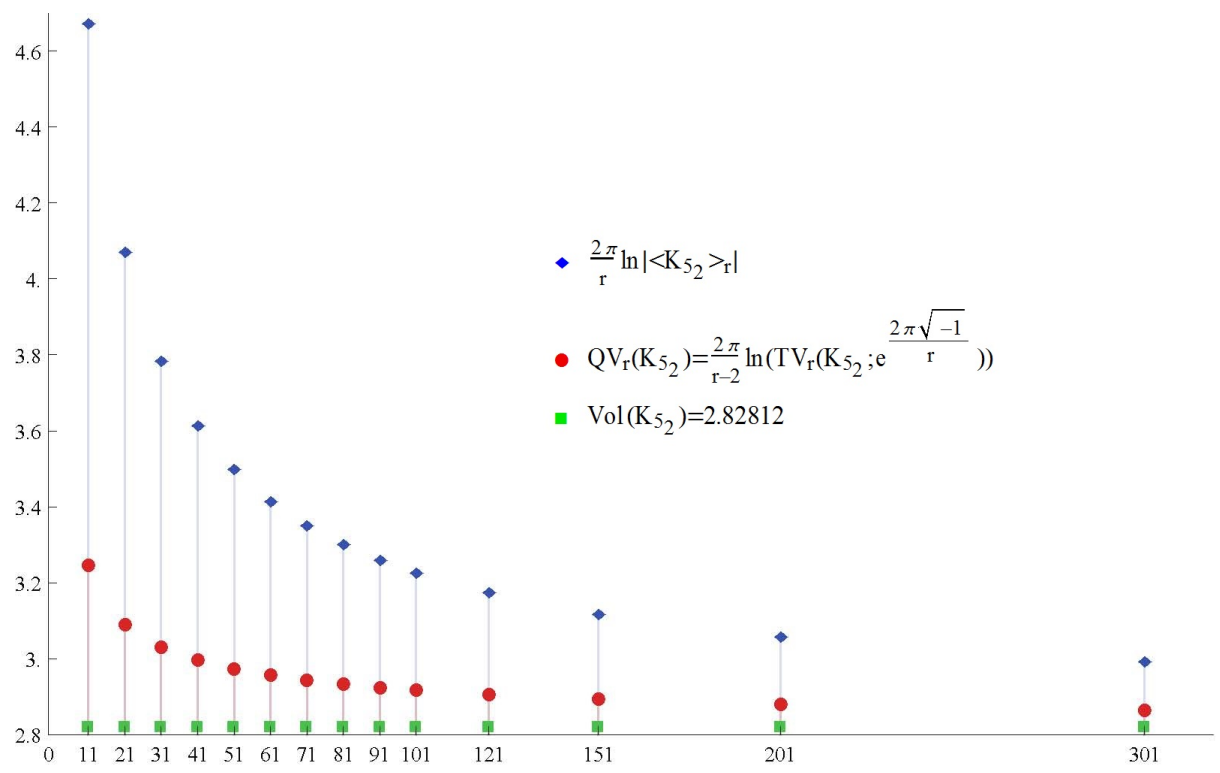

Figure 8. Comparison of different invariants for $K_{5_{2}}$

The manifold $M_{3_{6}}$ in the Callahan-Hildebrand-Weeks census [14] is also the complement of the $(-2,3,7)$-pretzel knot of Figure 9. It has the same volume as $S^{3} \backslash K_{5_{2}}$, namely

$$
\operatorname{vol}\left(M_{3_{6}}\right)=\operatorname{vol}\left(S^{3} \backslash K_{5_{2}}\right) \approx 2.82812 .
$$

According to Regina, $M_{36}$ can be represented by the ideal triangulation of Figure 10 . 


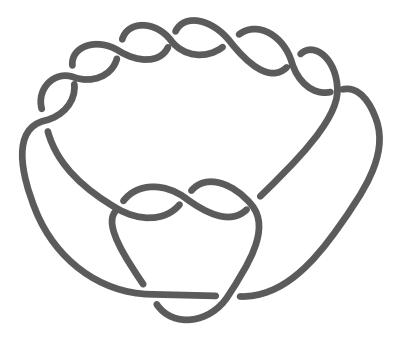

FIGURE 9. The $(-2,3,7)$-pretzel knot
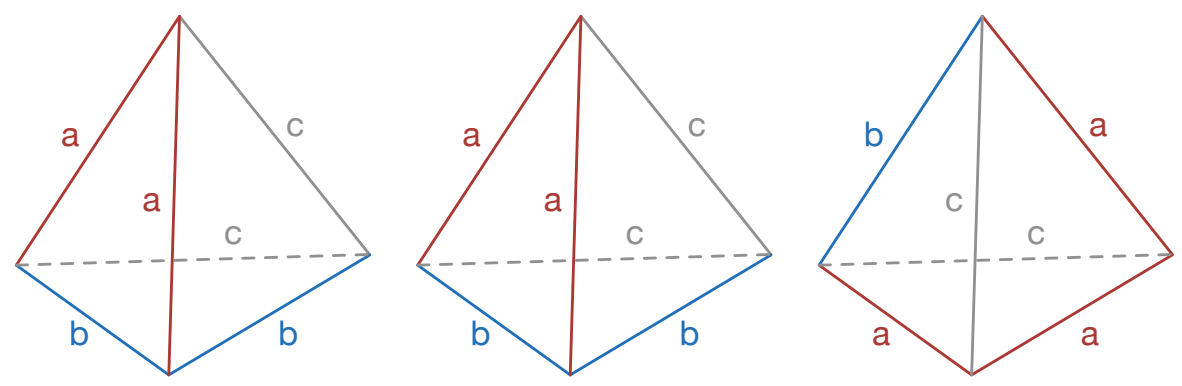

FIGURE 10.

Then for $r \geqslant 3$, we have

$$
\operatorname{TV}_{r}\left(M_{3_{6}}\right)=\sum_{(a, b, c) \in A_{r}} w_{a} w_{b} w_{c}\left|\begin{array}{lll}
a & a & b \\
b & c & c
\end{array}\right|\left|\begin{array}{lll}
a & a & b \\
b & c & c
\end{array}\right|\left|\begin{array}{lll}
a & b & c \\
a & a & c
\end{array}\right|,
$$

where $A_{r}$ consists of all triples $(a, b, c)$ of elements of $I_{r}$ such that $(a, a, b),(b, b, c),(c, c, a)$, $(a, b, c)$ and $(a, a, c)$ are admissible.

The table below shows a few values of $Q V_{r}\left(K_{5_{2}}\right)$ and $Q V_{r}\left(M_{3_{6}}\right)$. We observe that $Q V_{r}\left(K_{5_{2}}\right)$ and $Q V_{r}\left(M_{3_{6}}\right)$ are distinct, but are getting closer to each other and seem to converge to 2.82812 as $r$ grows.

\begin{tabular}{|c||c|c|c|}
\hline $\mathrm{r}$ & 9 & 11 & 21 \\
\hline$Q V_{r}\left(K_{5_{2}}\right)$ & 3.3239396087031623282 & 3.2528240712684816477 & 3.0958786489268195966 \\
\hline$Q V_{r}\left(M_{3_{6}}\right)$ & 3.2936286562299185780 & 3.2291939333749922011 & 3.0954357480831343159 \\
\hline $\mathrm{r}$ & 31 & 51 & 101 \\
\hline$Q V_{r}\left(K_{5_{2}}\right)$ & 3.0365668215995635907 & 2.9792536251826401549 & 2.9230944207585713174 \\
\hline$Q V_{r}\left(M_{3_{6}}\right)$ & 3.0365081953458580040 & 2.9792532229139281449 & 2.9230944207610719723 \\
\hline
\end{tabular}

The manifold $M_{38}$ in the Callahan-Hildebrand-Weeks census [14], which is not the complement of any knot in $S^{3}$, also has the same volume as $S^{3} \backslash K_{5_{2}}$. According to Regina, $M_{3_{8}}$ has an ideal triangulation that has the same colors as that of $S^{3} \backslash K_{5_{2}}$ drawn above. As a consequence, $Q V_{r}\left(M_{3_{8}}\right)$ coincides with $Q V_{r}\left(K_{5_{2}}\right)$ for all $r \geqslant 3$.

3.3. The $K_{61}$ knot complement. According to SnapPy [21] and Regina [12], the complement of the knot $K_{6_{1}}$ has volume

$$
\operatorname{vol}\left(S^{3} \backslash K_{6_{1}}\right) \approx 3.16396
$$


and can be described by the ideal triangulation of Figure 11.
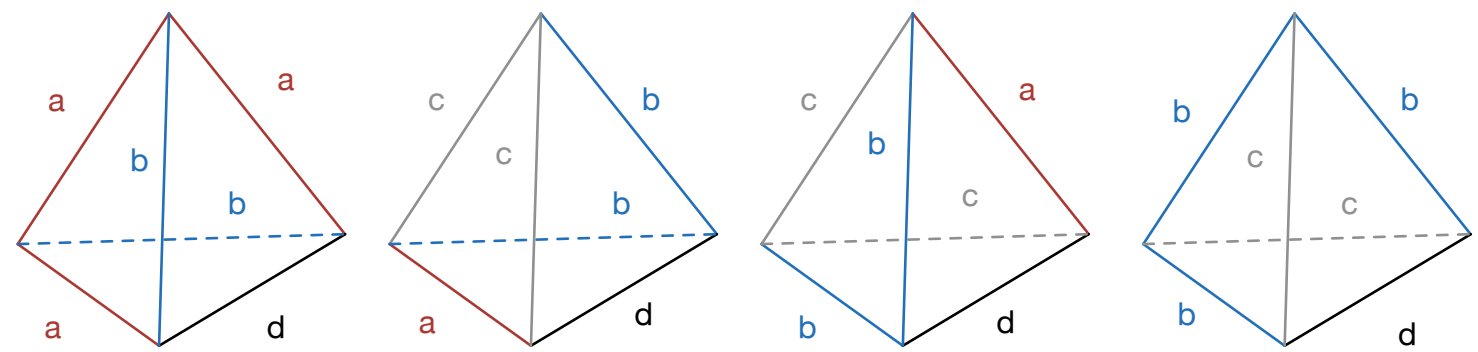

FIGURE 11.

This gives

$$
\operatorname{TV}_{r}\left(K_{6_{1}}\right)=\sum_{(a, b, c, d) \in A_{r}} w_{a} w_{b} w_{c} w_{d}\left|\begin{array}{lll}
a & a & b \\
a & d & b
\end{array}\right|\left|\begin{array}{lll}
a & c & c \\
b & b & d
\end{array}\right|\left|\begin{array}{lll}
b & b & c \\
a & c & d
\end{array}\right|\left|\begin{array}{lll}
b & b & c \\
b & d & c
\end{array}\right|,
$$

where $A_{r}$ consists of quadruples $(a, b, c, d)$ of elements of $I_{r}$ such that all the triples involved are admissible. From this, we have the following table of values of $Q V_{r}\left(K_{6_{1}}\right)$.

\begin{tabular}{|c||c|c|c|c|c|c|c|c|}
\hline $\mathrm{r}$ & 5 & 7 & 9 & 11 & 21 & 31 & 41 & 51 \\
\hline$Q V_{r}\left(K_{6_{1}}\right)$ & 3.83348 & 3.63472 & 3.46573 & 3.39987 & 3.34732 & 3.31699 & 3.29688 & 3.28214 \\
\hline$r$ & 61 & 71 & 81 & 91 & 101 & 121 & 151 & 201 \\
\hline \hline$Q V_{r}\left(K_{6_{1}}\right)$ & 3.27076 & 3.26165 & 3.25417 & 3.24790 & 3.24255 & 3.23390 & 3.22431 & 3.21353 \\
\hline
\end{tabular}

Figure 12 compares a few values of $Q V_{r}\left(K_{6_{1}}\right)$ with those of the Kashev invariant $\left\langle K_{6_{1}}\right\rangle_{r}$ and with the volume $\operatorname{vol}\left(S^{3} \backslash K_{6_{1}}\right)$.

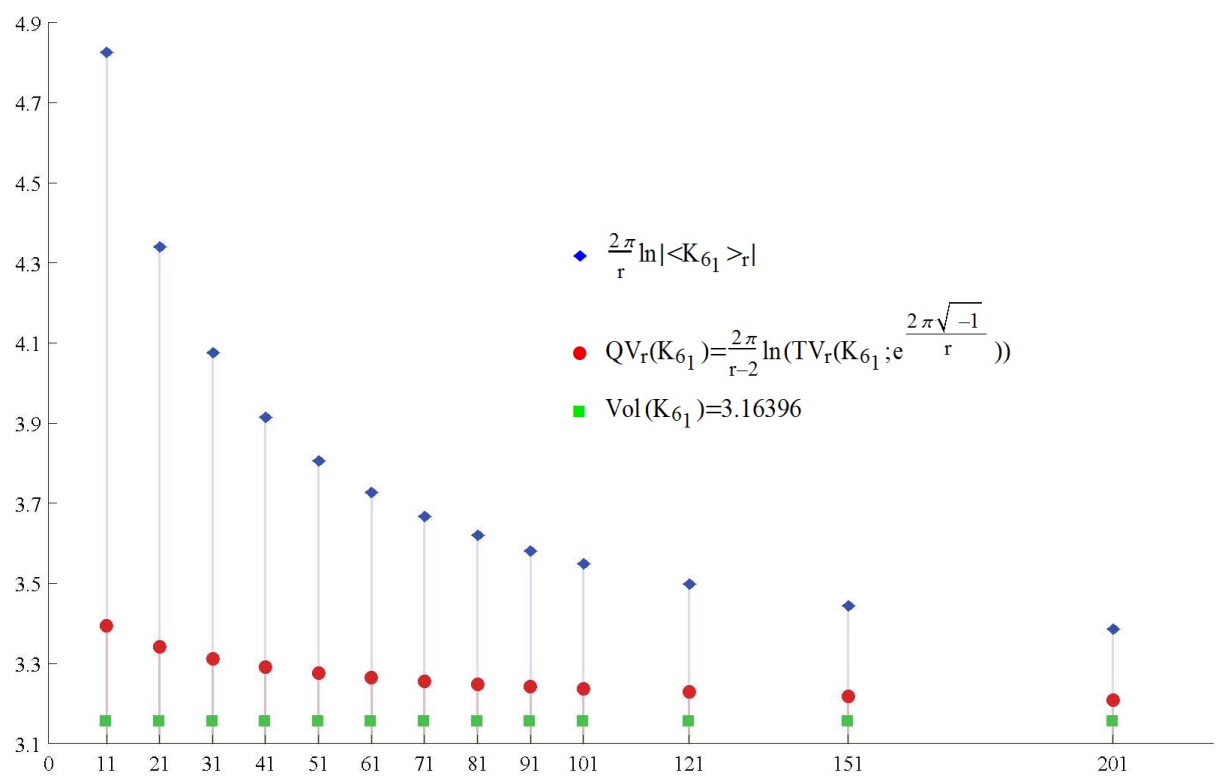

FIGURE 12. Comparison of different invariants for $K_{6_{1}}$ 


\subsection{Some non-orientable cusped 3-manifolds.}

3.4.1. The Gieseking manifold. The manifold $N_{1_{1}}$ in the Callahan-Hildebrand-Weeks census [14], also known as the Gieseking manifold, is the smallest non-orientable cusped 3-manifold. It has an ideal triangulation with a single tetrahedron which, by an Euler characteristic calculation, has only one edge. According to SnapPy [21] and Regina [12], the Gieseking manifold has volume

$$
\operatorname{vol}\left(N_{1_{1}}\right) \approx 1.01494 \text {. }
$$

By Definition 2.5, we have

$$
\mathrm{TV}_{r}\left(N_{1_{1}}\right)=\sum_{a \in A_{r}} w_{a}\left|\begin{array}{lll}
a & a & a \\
a & a & a
\end{array}\right|,
$$

where $A_{r}$ consist of integers $a$ such that $0 \leqslant a \leqslant\lfloor(r-2) / 3\rfloor$. Here \lfloor\rfloor is the floor function that $\lfloor x\rfloor$ equals the greatest integer less than or equal to $x$. From this, we have the following table of values of $Q V_{r}\left(N_{1_{1}}\right)$.

\begin{tabular}{|c||c|c|c|c|c|c|c|c|}
\hline $\mathrm{r}$ & 7 & 9 & 11 & 21 & 31 & 41 & 51 & 61 \\
\hline$Q V_{r}\left(N_{1_{1}}\right)$ & 1.81736 & 1.66782 & 1.62276 & 1.43255 & 1.33012 & 1.27064 & 1.23174 & 1.20411 \\
\hline$r$ & 71 & 81 & 91 & 101 & 201 & 301 & 401 & 501 \\
\hline$Q V_{r}\left(N_{1_{1}}\right)$ & 1.18335 & 1.16711 & 1.15401 & 1.14319 & 1.08943 & 1.06872 & 1.05748 & 1.05035 \\
\hline
\end{tabular}

3.4.2. Manifold $N_{2_{1}}$. According to SnapPy [21] and Regina [12], the manifold $N_{2_{1}}$ in CallahanHildebrand-Weeks census [14] has volume

$$
\operatorname{vol}\left(N_{2_{1}}\right) \approx 1.83193
$$

and has the following ideal triangulation.
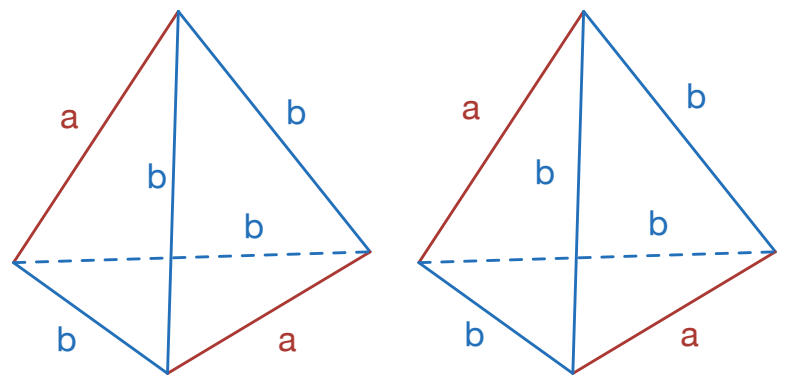

By Definition 2.5, we have

$$
\operatorname{TV}_{r}\left(N_{2_{1}}\right)=\sum_{(a, b) \in A_{r}} w_{a} w_{b}\left|\begin{array}{lll}
a & b & b \\
a & b & b
\end{array}\right|\left|\begin{array}{lll}
a & b & b \\
a & b & b
\end{array}\right|
$$

where $A_{r}$ consist of the pairs $(a, b)$ of elements of $I_{r}$ such that $(a, b, b)$ is admissible. From this, we have the following table of values of $Q V_{r}\left(N_{2_{1}}\right)$.

\begin{tabular}{|c||c|c|c|c|c|c|c|c|}
\hline $\mathrm{r}$ & 5 & 7 & 9 & 11 & 21 & 31 & 41 & 51 \\
\hline$Q V_{r}\left(N_{2_{1}}\right)$ & 2.90345 & 2.54929 & 2.46119 & 2.42036 & 2.20099 & 2.11235 & 2.06163 & 2.02810 \\
\hline$r$ & 61 & 71 & 81 & 91 & 101 & 121 & 151 & 201 \\
\hline$Q V_{r}\left(N_{2_{1}}\right)$ & 2.00403 & 1.98578 & 1.97140 & 1.95974 & 1.95006 & 1.93489 & 1.91876 & 1.90140 \\
\hline
\end{tabular}


3.5. Smallest hyperbolic 3-manifolds with totally geodesic boundary. By [39], any orientable hyperbolic 3-manifold $M_{\min }$ with non-empty totally geodesic boundary that has minimum volume admits a tetrahedral decomposition by two regular truncated hyperideal tetrahedra of dihedral angles $\pi / 6$. As a consequence, such a hyperbolic manifold has volume

$$
\operatorname{vol}\left(M_{\min }\right) \approx 6.452
$$

Such minimums are not unique and are classified in [23]. In particular, the boundary of each of them is a connected surface of genus 2, and an Euler characteristic calculation shows that each ideal triangulation of $M_{\min }$ with two tetrahedra has only one edge, as in Figure 13 .
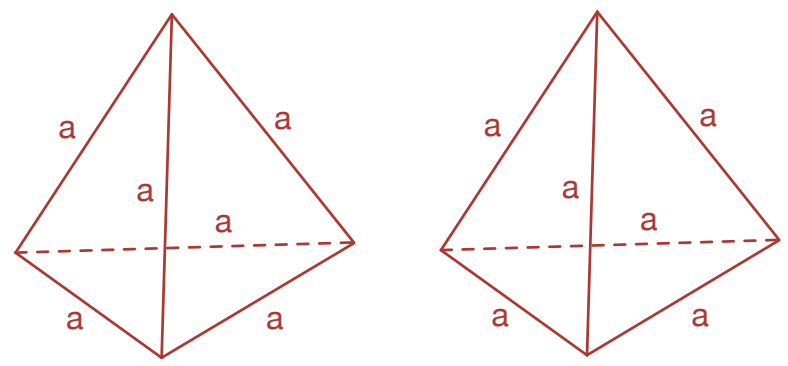

FIGURE 13.

Therefore, for $r \geqslant 3$,

$$
\operatorname{TV}_{r}\left(M_{\min }\right)=\sum_{a \in A_{r}} w_{a}\left|\begin{array}{lll}
a & a & a \\
a & a & a
\end{array}\right|\left|\begin{array}{ccc}
a & a & a \\
a & a & a
\end{array}\right|
$$

where $A_{r}$ consists of all integers $a$ with $0 \leqslant a \leqslant\lfloor(r-2) / 3\rfloor$. Here $\mathrm{TV}_{r}\left(M_{\min }\right)$ is negative for some values of $r$. In this case, we require the argument of the logarithm to be in the interval $[0,2 \pi)$, so that the imaginary part of $Q V_{r}\left(M_{\min }\right)=\frac{2 \pi}{r-2} \log \left(\mathrm{TV}_{r}\left(M_{\min }\right)\right)$ is either 0 or $2 \pi^{2} /(r-2)$. As a consequence, this imaginary part converges to 0 and it suffices to consider the real part to test the convergence of $Q V_{r}\left(M_{\min }\right)$.

We have the following table of the values of the real part $\Re\left(Q V_{r}\left(M_{\min }\right)\right)$ of $Q V_{r}\left(M_{\min }\right)$.

\begin{tabular}{|c||c|c|c|c|c|c|c|c|}
\hline$r$ & 11 & 21 & 31 & 41 & 51 & 61 & 71 & 81 \\
\hline$\Re\left(Q V_{r}\left(M_{\min }\right)\right)$ & 4.39782 & 5.12434 & 5.44590 & 5.63235 & 5.75566 & 5.84395 & 5.91063 & 5.96297 \\
\hline$r$ & 91 & 101 & 201 & 301 & 401 & 501 & 1001 & 2001 \\
\hline$\Re\left(Q V_{r}\left(M_{\min }\right)\right)$ & 6.00526 & 6.04022 & 6.21400 & 6.28075 & 6.31684 & 6.33970 & 6.38935 & 6.41741 \\
\hline
\end{tabular}

Figure 14 below illustrates the asymptotic behavior of $Q V_{r}\left(M_{\min }\right)$, where the dots represent the points $\left(r, Q V_{r}\left(M_{\min }\right)\right)$ and the squares represent the points $\left(r, \operatorname{vol}\left(M_{\min }\right)\right)$.

\section{EVIDENCE FOR CLOSED 3-MANIFOLDS}

We now consider Conjectures 1.1 and 1.2 in the case of closed manifolds ([55, 59, 61]), and provide evidence for these conjectures by numerically calculating the Reshetikhin-Turaev invariants of a few closed 3-manifolds obtained by doing Dehn surgeries along the knots $K_{4_{1}}$ and $K_{5_{2}}$. 


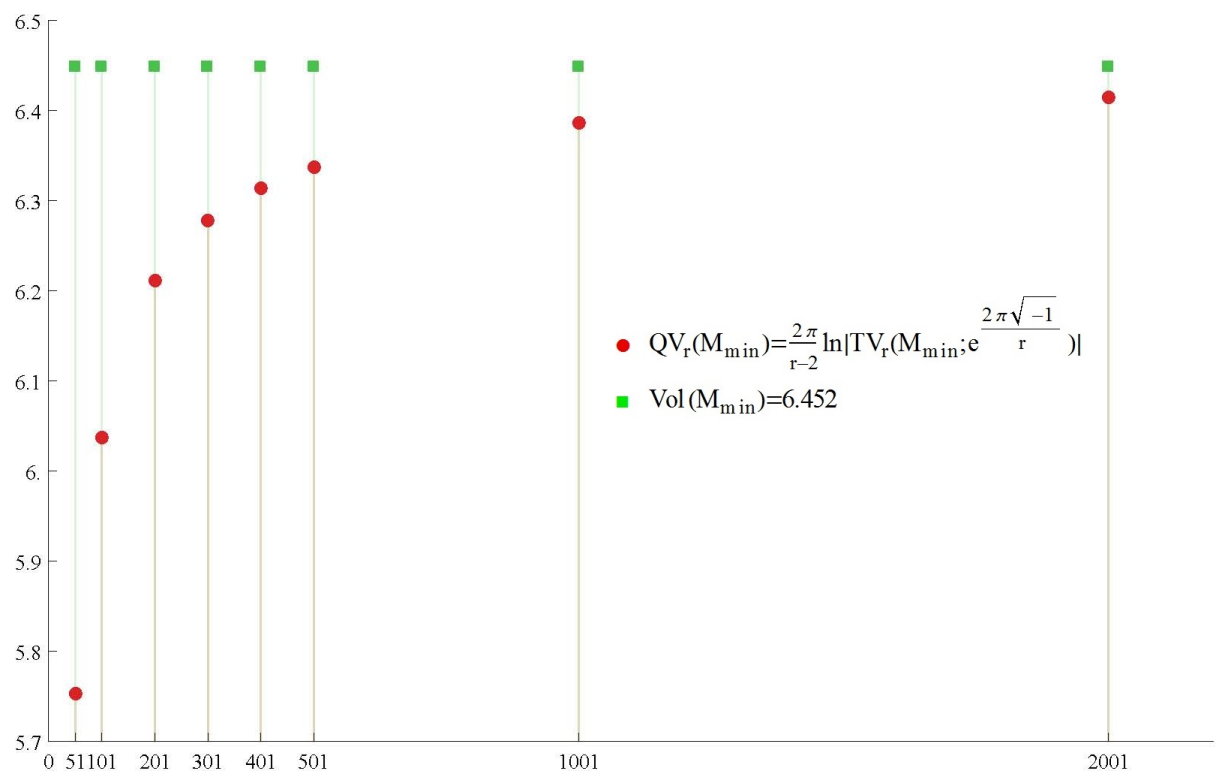

FIGURE 14. Asymptotics of $Q V_{r}\left(M_{\min }\right)$

According to [38], if $M$ is obtained from $S^{3}$ by doing a $p$-surgery along a knot $K$, then for an odd $r \geqslant 3$ the Reshetikhin-Turaev invariant $\operatorname{RT}_{r}(M ; q)$ of $M$ at $q=e^{\frac{2 \pi \sqrt{-1}}{r}}$ is calculated as (4.1)

$$
\mathrm{RT}_{r}(M ; q)=\frac{2}{r} e^{-\epsilon(p)\left(-\frac{3}{r}-\frac{r+1}{4}\right) \pi \sqrt{-1}}\left(\sum_{n=0}^{r-2}\left(\sin \frac{2(n+1) \pi}{r}\right)^{2}\left(-e^{\frac{\pi \sqrt{-1}}{r}}\right)^{p\left(n^{2}+2 n\right)} J_{n+1}\left(K ; e^{\frac{4 \pi \sqrt{-1}}{r}}\right)\right),
$$

where $\epsilon(p)$ is the sign of $p$, and $J_{n}\left(K ; e^{\frac{4 \pi \sqrt{-1}}{r}}\right)$ is the value of the $n$-th colored Jones polynomial $J_{n}(K ; t)$ of $K$ at $t=e^{\frac{4 \pi \sqrt{-1}}{r}}$, normalized in such a way that $J_{n}($ unknot $)=1$.

Remark 4.1. The conventions in skein theory ([32, 11, 38]) make use of a variable $A$ that is, either a primitive $2 r$-th root of unity for an integer $r$, or a primitive $r$-th root of unity for an odd integer $r$. The root of unity $q$ in the definition of the Turaev-Viro invariant then corresponds to $A^{2}$, while the variable $t$ of the colored Jones polynomial corresponds to $A^{4}$. Formula (4.1) deals with the case where $A=e^{\frac{\pi \sqrt{-1}}{r}}$ for $r$ odd, in which case $q=e^{\frac{2 \pi \sqrt{-1}}{r}}$ and $t=e^{\frac{4 \pi \sqrt{-1}}{r}}$.

Remark 4.2. Formula (4.1) is directly derived from [38, §4.1]. In Lickorish's notation and letting $A=e^{\frac{\pi \sqrt{-1}}{r}}$, one has $\mu=\frac{1}{\sqrt{r}} \sin \frac{2 \pi}{r},\langle\mu \omega\rangle_{U_{-}}^{\sigma}=e^{-\epsilon(p)\left(-\frac{3}{r}-\frac{r+1}{4}\right) \pi \sqrt{-1}},\langle\mu \omega\rangle_{U}^{-1}=\frac{2}{\sqrt{r}} \sin \frac{2 \pi}{r}$, and for $K_{p}$ the knot $K$ with framing $p$,

$$
\langle\omega\rangle_{K_{p}}=\sum_{n=0}^{r-2}\left(\frac{\sin \frac{2(n+1) \pi}{r}}{\sin \frac{2 \pi}{r}}\right)^{2}\left(-e^{\frac{\pi \sqrt{-1}}{r}}\right)^{p\left(n^{2}+2 n\right)} J_{n+1}\left(K ; e^{\frac{4 \pi \sqrt{-1}}{r}}\right) .
$$

Multiplying the above terms together, one gets formula 4.1).

To calculate the growth rate of $\operatorname{RT}_{r}(M ; q)$ as $r$ approaches infinity, it is equivalent to calculate the limit of the following quantity

$$
Q_{r}(M)=2 \pi \sqrt{-1} \log \left(\operatorname{RT}_{r}\left(M ; e^{\frac{2 \pi \sqrt{-1}}{r}}\right) / \operatorname{RT}_{r-2}\left(M ; e^{\frac{2 \pi \sqrt{-1}}{r-2}}\right)\right),
$$

where the logarithm log is chosen so that its imaginary part lies in the interval $(-\pi, \pi)$. 
4.1. Surgeries along the figure-eight knot. In this subsection, we denote by $M_{p}$ the manifold obtained from $S^{3}$ by doing a $p$-surgery along the figure-eight knot $K_{4_{1}}$. Recall from [56] that $M_{p}$ is hyperbolic if and only if $|p| \geqslant 5$. By [43], the $n$-th colored Jones polynomial of $K_{4_{1}}$ equals

$$
J_{n}\left(K_{4_{1}}, t\right)=\sum_{k=0}^{n-1} \prod_{i=1}^{k}\left(t^{\frac{n-i}{2}}-t^{-\frac{n-i}{2}}\right)\left(t^{\frac{n+i}{2}}-t^{-\frac{n+i}{2}}\right) .
$$

In the tables below, we list the values of $Q_{r}\left(M_{p}\right)$ modulo $\pi^{2} \mathbb{Z}$ for $p=-6,-5,5,6,7,8$ and for $r=51,101,151,201,301$ and 501 .

4.1.1. $p=-6$. According to SnapPy [21],

$$
\mathrm{CS}\left(M_{-6}\right)+\operatorname{vol}\left(M_{-6}\right) \sqrt{-1}=-1.34092+1.28449 \sqrt{-1} \bmod \pi^{2} \mathbb{Z},
$$

and by (4.1) and (4.2), we have

\begin{tabular}{|c||c|c|c|}
\hline $\mathrm{r}$ & 51 & 101 & 151 \\
\hline$Q_{r}\left(M_{-6}\right)$ & $-1.34241+1.22717 \sqrt{-1}$ & $-1.32879+1.28425 \sqrt{-1}$ & $-1.33549+1.28440 \sqrt{-1}$ \\
\hline $\mathrm{r}$ & 201 & 301 & 501 \\
\hline$Q_{r}\left(M_{-6}\right)$ & $-1.33786+1.28443 \sqrt{-1}$ & $-1.33956+1.28446 \sqrt{-1}$ & $-1.34043+1.28448 \sqrt{-1}$ \\
\hline
\end{tabular}

4.1.2. $p=-5$. According to SnapPy [21],

$$
\mathrm{CS}\left(M_{-5}\right)+\operatorname{vol}\left(M_{-5}\right) \sqrt{-1}=-1.52067+0.98137 \sqrt{-1} \bmod \pi^{2} \mathbb{Z},
$$

and by (4.1) and (4.2), we have

\begin{tabular}{|c||c|c|c|}
\hline $\mathrm{r}$ & 51 & 101 & 151 \\
\hline$Q_{r}\left(M_{-5}\right)$ & $-1.50445+0.87410 \sqrt{-1}$ & $-1.51521+0.98003 \sqrt{-1}$ & $-1.51712+0.98130 \sqrt{-1}$ \\
\hline $\mathrm{r}$ & 201 & 301 & 501 \\
\hline$Q_{r}\left(M_{-5}\right)$ & $-1.51865+0.98131 \sqrt{-1}$ & $-1.51977+0.98134 \sqrt{-1}$ & $-1.52035+0.98136 \sqrt{-1}$ \\
\hline
\end{tabular}

4.1.3. $p=5$. According to SnapPy [21],

$$
\operatorname{CS}\left(M_{5}\right)+\operatorname{vol}\left(M_{5}\right) \sqrt{-1}=1.52067+0.98137 \sqrt{-1} \bmod \pi^{2} \mathbb{Z},
$$

and by (4.1) and (4.2), we have

\begin{tabular}{|c||c|c|c|}
\hline $\mathrm{r}$ & 51 & 101 & 151 \\
\hline$Q_{r}\left(M_{5}\right)$ & $1.50445+0.87410 \sqrt{-1}$ & $1.51521+0.98003 \sqrt{-1}$ & $1.51712+0.98130 \sqrt{-1}$ \\
\hline $\mathrm{r}$ & 201 & 301 & 501 \\
\hline$Q_{r}\left(M_{5}\right)$ & $1.51865+0.98131 \sqrt{-1}$ & $1.51977+0.98134 \sqrt{-1}$ & $1.52035+0.98136 \sqrt{-1}$ \\
\hline
\end{tabular}


4.1.4. $p=6$. According to SnapPy [21],

$$
\mathrm{CS}\left(M_{6}\right)+\operatorname{vol}\left(M_{-6}\right) \sqrt{-1}=1.34092+1.28449 \sqrt{-1} \bmod \pi^{2} \mathbb{Z},
$$

and by (4.1) and (4.2), we have

\begin{tabular}{|c||c|c|c|}
\hline $\mathrm{r}$ & 51 & 101 & 151 \\
\hline$Q_{r}\left(M_{6}\right)$ & $1.34241+1.22717 \sqrt{-1}$ & $1.32879+1.28425 \sqrt{-1}$ & $1.33549+1.28440 \sqrt{-1}$ \\
\hline \hline $\mathrm{r}$ & 201 & 301 & 501 \\
\hline$Q_{r}\left(M_{6}\right)$ & $1.33786+1.28443 \sqrt{-1}$ & $1.33956+1.28446 \sqrt{-1}$ & $1.34043+1.28448 \sqrt{-1}$ \\
\hline
\end{tabular}

4.1.5. $p=7$. According to SnapPy [21],

$$
\operatorname{CS}\left(M_{7}\right)+\operatorname{vol}\left(M_{7}\right) \sqrt{-1}=1.19653+1.46378 \sqrt{-1} \bmod \pi^{2} \mathbb{Z},
$$

and by (4.1) and (4.2), we have

\begin{tabular}{|c||c|c|c|}
\hline $\mathrm{r}$ & 51 & 101 & 151 \\
\hline$Q_{r}\left(M_{7}\right)$ & $1.10084+1.43670 \sqrt{-1}$ & $1.18016+1.46354 \sqrt{-1}$ & $1.18930+1.46367 \sqrt{-1}$ \\
\hline $\mathrm{r}$ & 201 & 301 & 501 \\
\hline$Q_{r}\left(M_{7}\right)$ & $1.19246+1.46372 \sqrt{-1}$ & $1.19472+1.46375 \sqrt{-1}$ & $1.19588+1.46377 \sqrt{-1}$ \\
\hline
\end{tabular}

4.1.6. $p=8$. According to SnapPy [21],

$$
\mathrm{CS}\left(M_{8}\right)+\operatorname{vol}\left(M_{8}\right) \sqrt{-1}=1.07850+1.58317 \sqrt{-1} \bmod \pi^{2} \mathbb{Z},
$$

and by (4.1) and (4.2), we have

\begin{tabular}{|c||c|c|c|}
\hline $\mathrm{r}$ & 51 & 101 & 151 \\
\hline$Q_{r}\left(M_{8}\right)$ & $0.96311+1.57167 \sqrt{-1}$ & $1.05821+1.58282 \sqrt{-1}$ & $1.06949+1.58304 \sqrt{-1}$ \\
\hline $\mathrm{r}$ & 201 & 301 & 501 \\
\hline$Q_{r}\left(M_{8}\right)$ & $1.07343+1.58309 \sqrt{-1}$ & $1.07625+1.58313 \sqrt{-1}$ & $1.07769+1.58315 \sqrt{-1}$ \\
\hline
\end{tabular}

4.2. Surgeries along $K_{5_{2}}$. In this subsection, we let $M_{p}$ be the manifold obtained from $S^{3}$ by doing a $p$-surgery along the knot $K_{5_{2}}$. Recall that $M_{p}$ is hyperbolic if and only if $p \leqslant-1$ or $p \geqslant 5$. By [40], the $n$-th colored Jones polynomial of $K_{5_{2}}$ is equal to

$$
J_{n}\left(K_{5_{2}}, t\right)=\sum_{k=0}^{n-1} t^{-\frac{k(k+3)}{4}} c_{k} \prod_{i=1}^{k}\left(t^{\frac{n-i}{2}}-t^{-\frac{n-i}{2}}\right)\left(t^{\frac{n+i}{2}}-t^{-\frac{n+i}{2}}\right)
$$

where

$$
c_{k}=(-1)^{k} t^{-\frac{5 k^{2}+7 k}{4}} \sum_{i=0}^{k} t^{-\frac{i^{2}-2 i-3 k i}{2}} \frac{[k] !}{[i] ![k-i] !} .
$$

Here the formula differs from that of [40] by replacing $t$ with $t^{-1}$. This comes from the chirality of $K_{5_{2}}$. Here we stick to the convention that is used in SnapPy [21], which is the mirror image of the one used in [40].

In the tables below, we list the values of $Q_{r}\left(M_{p}\right)$ modulo $\pi^{2} \mathbb{Z}$ for $p=-3,-2,-1,5,6,7$ and for $r=51,75,101,125,151$ and 201. 
4.2.1. $p=-3$. According to SnapPy [21],

$$
\mathrm{CS}\left(M_{-3}\right)+\operatorname{vol}\left(M_{-3}\right) \sqrt{-1}=-4.45132+2.10310 \sqrt{-1} \bmod \pi^{2} \mathbb{Z},
$$

and by (4.1) and (4.3), we have

\begin{tabular}{|c||c|c|c|}
\hline $\mathrm{r}$ & 51 & 75 & 101 \\
\hline$Q_{r}\left(M_{-3}\right)$ & $-4.37951+2.10038 \sqrt{-1}$ & $-4.41819+2.10200 \sqrt{-1}$ & $-4.43323+2.10247 \sqrt{-1}$ \\
\hline $\mathrm{r}$ & 125 & 151 & 201 \\
\hline$Q_{r}\left(M_{-3}\right)$ & $-4.43957+2.10268 \sqrt{-1}$ & $-4.44329+2.10281 \sqrt{-1}$ & $-4.44681+2.10293 \sqrt{-1}$ \\
\hline
\end{tabular}

4.2.2. $p=-2$. According to SnapPy [21],

$$
\mathrm{CS}\left(M_{-2}\right)+\operatorname{vol}\left(M_{-2}\right) \sqrt{-1}=-4.63884+1.84359 \sqrt{-1} \bmod \pi^{2} \mathbb{Z},
$$

and by (4.1) and (4.3), we have

\begin{tabular}{|c||c|c|c|}
\hline $\mathrm{r}$ & 51 & 75 & 101 \\
\hline$Q_{r}\left(M_{-2}\right)$ & $-4.59073+1.84822 \sqrt{-1}$ & $-4.61357+1.84289 \sqrt{-1}$ & $-4.62490+1.84317 \sqrt{-1}$ \\
\hline $\mathrm{r}$ & 125 & 151 & 201 \\
\hline$Q_{r}\left(M_{-2}\right)$ & $-4.62978+1.84331 \sqrt{-1}$ & $-4.63265+1.84339 \sqrt{-1}$ & $-4.63536+1.84348 \sqrt{-1}$ \\
\hline
\end{tabular}

\subsection{3. $p=-1$. According to SnapPy [21],}

$$
\mathrm{CS}\left(M_{-1}\right)+\operatorname{vol}\left(M_{-1}\right) \sqrt{-1}=-4.86783+1.39851 \sqrt{-1} \bmod \pi^{2} \mathbb{Z},
$$

and by (4.1) and (4.3), we have

\begin{tabular}{|c||c|c|c|}
\hline $\mathrm{r}$ & 51 & 75 & 101 \\
\hline$Q_{r}\left(M_{-1}\right)$ & $-4.84865+1.40943 \sqrt{-1}$ & $-4.85045+1.39808 \sqrt{-1}$ & $-4.85817+1.39817 \sqrt{-1}$ \\
\hline $\mathrm{r}$ & 125 & 151 & 201 \\
\hline$Q_{r}\left(M_{-1}\right)$ & $-4.86157+1.39827 \sqrt{-1}$ & $-4.86355+1.39834 \sqrt{-1}$ & $-4.86542+1.39841 \sqrt{-1}$ \\
\hline
\end{tabular}

4.2.4. $p=5$. According to SnapPy [21],

$$
\mathrm{CS}\left(M_{5}\right)+\operatorname{vol}\left(M_{5}\right) \sqrt{-1}=-1.52067+0.98137 \sqrt{-1} \bmod \pi^{2} \mathbb{Z},
$$

and by (4.1) and (4.3), we have

\begin{tabular}{|c||c|c|c|}
\hline $\mathrm{r}$ & 51 & 75 & 101 \\
\hline$Q_{r}\left(M_{5}\right)$ & $-1.50445+0.87410 \sqrt{-1}$ & $-1.48899+0.96890 \sqrt{-1}$ & $-1.51521+0.98003 \sqrt{-1}$ \\
\hline $\mathrm{r}$ & 125 & 151 & 201 \\
\hline$Q_{r}\left(M_{5}\right)$ & $-1.51539+0.98098 \sqrt{-1}$ & $-1.51712+0.98130 \sqrt{-1}$ & $-1.51865+0.98131 \sqrt{-1}$ \\
\hline
\end{tabular}


4.2.5. $p=6$. According to SnapPy [21],

$$
\operatorname{CS}\left(M_{6}\right)+\operatorname{vol}\left(M_{6}\right) \sqrt{-1}=-1.51206+1.41406 \sqrt{-1} \bmod \pi^{2} \mathbb{Z},
$$

and by (4.1) and (4.3), we have

\begin{tabular}{|c||c|c|c|}
\hline $\mathrm{r}$ & 51 & 75 & 101 \\
\hline$Q_{r}\left(M_{6}\right)$ & $-1.46756+1.40044 \sqrt{-1}$ & $-1.50631+1.41501 \sqrt{-1}$ & $-1.50836+1.41339 \sqrt{-1}$ \\
\hline $\mathrm{r}$ & 125 & 151 & 201 \\
\hline$Q_{r}\left(M_{6}\right)$ & $-1.50968+1.41356 \sqrt{-1}$ & $-1.51042+1.41372 \sqrt{-1}$ & $-1.51113+1.41386 \sqrt{-1}$ \\
\hline
\end{tabular}

4.2.6. $p=7$. According to SnapPy [21],

$$
\operatorname{CS}\left(M_{7}\right)+\operatorname{vol}\left(M_{7}\right) \sqrt{-1}=-1.55255+1.75713 \sqrt{-1} \bmod \pi^{2} \mathbb{Z},
$$

and by (4.1) and (4.3), we have

\begin{tabular}{|c||c|c|c|}
\hline $\mathrm{r}$ & 51 & 75 & 101 \\
\hline$Q_{r}\left(M_{7}\right)$ & $-1.53822+1.75178 \sqrt{-1}$ & $-1.55297+1.75315 \sqrt{-1}$ & $-1.55265+1.75507 \sqrt{-1}$ \\
\hline $\mathrm{r}$ & 125 & 151 & 201 \\
\hline$Q_{r}\left(M_{7}\right)$ & $-1.55257+1.75582 \sqrt{-1}$ & $-1.55255+1.75625 \sqrt{-1}$ & $-1.55254+1.75664 \sqrt{-1}$ \\
\hline
\end{tabular}

\section{AN INTEGRALITY CONJECTURE FOR TORUS LINK COMPLEMENTS}

In this section, we study the Turaev-Viro invariants for torus link complements. We propose the following Integrality Conjecture 5.1, and provide evidence by both rigorous ( $\$ 5.1$ and numerical (\$5.2) calculations.

Conjecture 5.1. Let $T_{(m, n)}$ be the $(m, n)$-torus link in $S^{3}$. If $r$ is relatively prime to $m$ and $n$, then $\mathrm{TV}_{r}\left(S^{3} \backslash T_{(m, n)}\right)$ is an integer independent of the choice of the roots of unity $q$.

5.1. Calculations for some torus links. In this subsection, we will rigorously calculate $\operatorname{TV}_{r}(M)$ for the complements of the unknot, the trefoil knot, the Hopf link and the torus links $T_{(2,4)}$ and $T_{(2,6)}$. As in the previous sections, for a link $L$ in $S^{3}$ we let

$$
\operatorname{TV}_{r}(L)=\operatorname{TV}_{r}\left(S^{3} \backslash L\right)
$$

All the ideal triangulations used in this section are obtained by using Regina [12] and SnapPy [21], and for simplicity, we will omit the arrows on the edges and keep only the colors.

\subsubsection{The unknot.}

Proposition 5.2. Let $U$ be the unknot in $S^{3}$. Then

$$
\mathrm{TV}_{r}(U)=1
$$

for all $r \geqslant 3$ and for all $q \in \mathbb{C}$ such that $q^{2}$ is a primitive root of unity of degree $r$. 

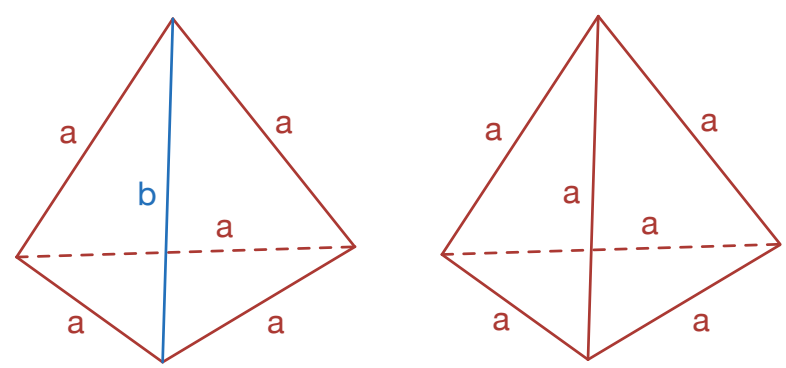

FIGURE 15.

Proof. The complement of the unknot admits the ideal triangulation represented in Figure 15 .

Therefore, for each $r \geqslant 3$, we have

$$
\begin{aligned}
\operatorname{TV}_{r}(U) & =\sum_{a, b} w_{a} w_{b}\left|\begin{array}{lll}
a & a & a \\
a & a & b
\end{array}\right|\left|\begin{array}{lll}
a & a & a \\
a & a & a
\end{array}\right| \\
& =\sum_{a} w_{a}\left|\begin{array}{lll}
a & a & a \\
a & a & a
\end{array}\right|\left(\sum_{b} w_{b}\left|\begin{array}{lll}
a & a & a \\
a & a & b
\end{array}\right|\right),
\end{aligned}
$$

where in the first row $(a, b) \in I_{r} \times I_{r}$ runs over all the admissible colorings at level $r$, and in the second row $a$ is over all the elements of $I_{r}$ such that $(a, a, a)$ is admissible and $b$ is over all elements of $I_{r}$ such that $(a, a, b)$ is admissible. Then the result follows from the following identity

$$
\sum_{b} w_{b}\left|\begin{array}{lll}
a & a & a \\
a & a & b
\end{array}\right|=\delta_{0, a} .
$$

To prove (5.1), we use the Orthogonality Property. Letting $m=0, s=b$ and $i=j=k=l=$ $n=a$ in 2.1 , we have

$$
\sum_{b} w_{b} w_{0}\left|\begin{array}{lll}
a & a & 0 \\
a & a & b
\end{array}\right|\left|\begin{array}{lll}
a & a & a \\
a & a & b
\end{array}\right|=\delta_{0, a},
$$

where $b$ is over all elements of $I_{r}$ such that $(a, b)$ is admissible at level $r$. Since $w_{0}=1$ and $\left|\begin{array}{lll}a & a & 0 \\ a & a & b\end{array}\right|=\frac{1}{[2 a+1]}$, we have

$$
\sum_{b} w_{b}\left|\begin{array}{ccc}
a & a & a \\
a & a & b
\end{array}\right|=[2 a+1] \cdot \delta_{0, a}=\delta_{0, a}
$$

Conjecture 5.3. Let $K$ be a knot in $S^{3}$. Then $\operatorname{TV}_{r}(K)=1$ for all $r \geqslant 3$ and for all $q \in \mathbb{C}$ such that $q^{2}$ is a primitive root of unity of degree $r$ if and only if $K=U$.

Remark 5.4. It is interesting to know whether there is an $M \neq S^{3} \backslash U$, not necessarily a link complement, such that $\mathrm{TV}_{r}(M)=1$ for all $r$ and $q$.

\subsubsection{The trefoil knot.}

Proposition 5.5. Let $T_{(2,3)}$ be the trefoil knot in $S^{3}$. Then

$$
\mathrm{TV}_{r}\left(T_{(2,3)}\right)=\left\lfloor\frac{r-2}{3}\right\rfloor+1
$$


for all $r \geqslant 3$ and for all $q \in \mathbb{C}$ such that $q^{2}$ is a primitive root of unity of degree $r$.

Proof. The complement of trefoil knot $T_{(2,3)}$ admits the ideal triangulation represented in Figure 16.
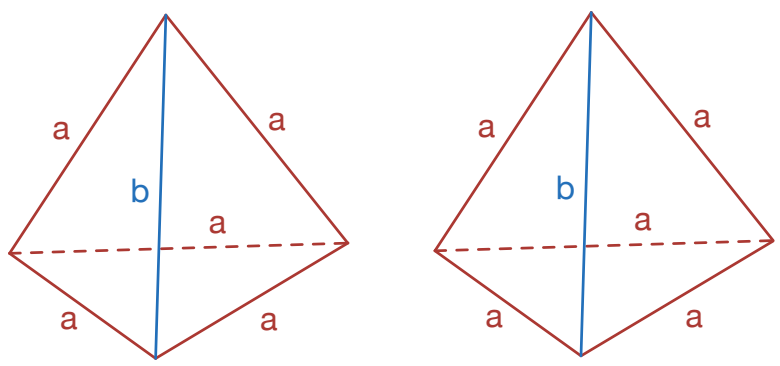

FIGURE 16.

Therefore, for each $r \geqslant 3$, we have

$$
\operatorname{TV}_{r}\left(T_{(2,3)}\right)=\sum_{a, b} w_{a} w_{b}\left|\begin{array}{lll}
a & a & a \\
a & a & b
\end{array}\right|\left|\begin{array}{lll}
a & a & a \\
a & a & b
\end{array}\right|,
$$

where $(a, b) \in I_{r} \times I_{r}$ runs over all the admissible colorings at level $r$. The triple $(a, a, a)$ being admissible implies that $a \in \mathbb{Z}$ and $a \leqslant(r-2) / 3$. Hence the right hand side equals

$$
\sum_{0 \leqslant a \leqslant \frac{r-2}{3}}\left(\sum_{b} w_{b} w_{a}\left|\begin{array}{lll}
a & a & a \\
a & a & b
\end{array}\right|\left|\begin{array}{ccc}
a & a & a \\
a & a & b
\end{array}\right|\right),
$$

where $a$ is over all the integers in that range and $b$ is over all elements of $I_{r}$ such that $(a, a, b)$ is admissible. Letting $i=j=k=l=m=n=a$ and $s=b$ in the Orthogonality Property (2.1), we have

$$
\sum_{b} w_{b} w_{a}\left|\begin{array}{lll}
a & a & a \\
a & a & b
\end{array}\right|\left|\begin{array}{ccc}
a & a & a \\
a & a & b
\end{array}\right|=1,
$$

where $b$ is over all elements of $I_{r}$ such that $(a, a, b)$ is admissible. As a consequence,

$$
\operatorname{TV}_{r}\left(T_{(2,3)}\right)=\sum_{0 \leqslant a \leqslant \frac{r-2}{3}} 1=\left\lfloor\frac{r-2}{3}\right\rfloor+1
$$

5.1.3. The Hopf link and torus links $T_{(2,4)}$ and $T_{(2,6)}$.

Proposition 5.6. Let $T_{(2,2)}$ be the Hopf link in $S^{3}$. Then

$$
\mathrm{TV}_{r}\left(T_{(2,2)}\right)=r-1
$$

for all $r \geqslant 3$ and for all $q \in \mathbb{C}$ such that $q^{2}$ is a primitive root of unity of degree $r$.

Proof. The complement of the Hopf link admits the ideal triangulation represented in Figure 17 

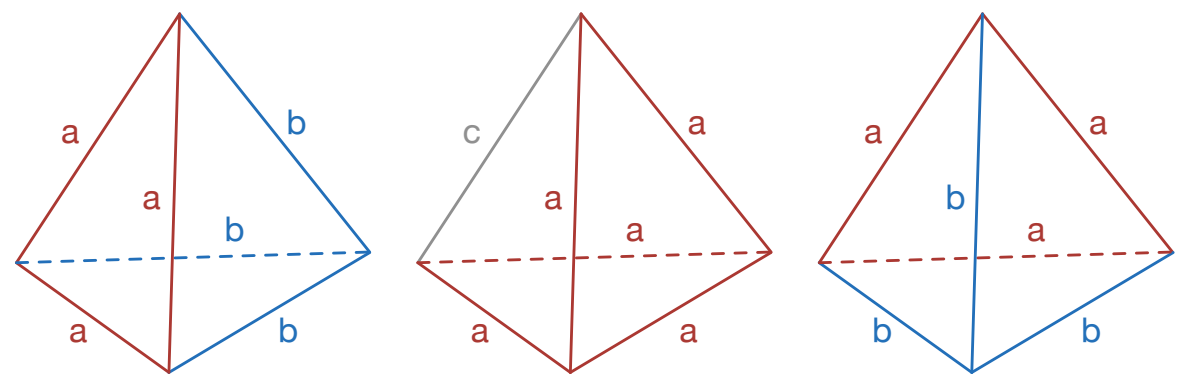

FIGURE 17.

Therefore, for each $r \geqslant 3$, we have

$$
\begin{aligned}
\operatorname{TV}_{r}\left(T_{(2,2)}\right) & =\sum_{a, b, c} w_{a} w_{b} w_{c}\left|\begin{array}{lll}
a & a & a \\
a & a & c
\end{array}\right|\left|\begin{array}{lll}
a & a & a \\
b & b & b
\end{array}\right|\left|\begin{array}{lll}
a & a & a \\
b & b & b
\end{array}\right| \\
& =\sum_{a, b} w_{a} w_{b}\left|\begin{array}{lll}
a & a & a \\
b & b & b
\end{array}\right|\left|\begin{array}{lll}
a & a & a \\
b & b & b
\end{array}\right|\left(\sum_{c} w_{c}\left|\begin{array}{lll}
a & a & a \\
a & a & c
\end{array}\right|\right),
\end{aligned}
$$

where in the first row $(a, b, c)$ runs over all the admissible colorings at level $r$, and in the second row $c$ runs over all elements of $I_{r}$ such that all the involved quantum $6 j$-symbols are admissible. By (5.1), we have

$$
\sum_{c} w_{c}\left|\begin{array}{lll}
a & a & a \\
a & a & c
\end{array}\right|=\delta_{0, a}
$$

Therefore,

$$
\operatorname{TV}_{r}\left(T_{(2,2)}\right)=\sum_{b} w_{0} w_{b}\left|\begin{array}{lll}
0 & 0 & 0 \\
b & b & b
\end{array}\right|\left|\begin{array}{lll}
0 & 0 & 0 \\
b & b & b
\end{array}\right|=\sum_{b} 1,
$$

where $b$ is over all the elements in $I_{r}$ such that $(0, b, b)$ is admissible. Since this holds for all elements $b$ in $I_{r}$,

$$
\mathrm{TV}_{r}\left(T_{(2,2)}\right)=\left|I_{r}\right|=r-1 .
$$

Proposition 5.7. Let $T_{(2,4)}$ be the $(2,4)$-torus link in $S^{3}$. Then

$$
\operatorname{TV}_{r}\left(T_{(2,4)}\right)=\left(\left\lfloor\frac{r-2}{2}\right\rfloor+1\right)\left(\left\lfloor\frac{r-1}{2}\right\rfloor+1\right)
$$

for all $r \geqslant 3$ and for all $q \in \mathbb{C}$ such that $q^{2}$ is a primitive root of unity of degree $r$.

Proof. The complement of the torus link $T_{(2,4)}$ has the following ideal triangulation represented in Figure 18.

Therefore, for each $r \geqslant 3$, we have

$$
\begin{aligned}
\mathrm{TV}_{r}\left(T_{(2,4)}\right) & =\sum_{(a, b, c, d) \in A_{r}} w_{a} w_{b} w_{c} w_{d}\left|\begin{array}{lll}
a & a & b \\
c & c & c
\end{array}\right|\left|\begin{array}{lll}
a & a & b \\
c & c & c
\end{array}\right|\left|\begin{array}{lll}
b & b & b \\
a & a & d
\end{array}\right|\left|\begin{array}{lll}
b & b & b \\
a & a & a
\end{array}\right| \\
& =\sum_{a, b, c} w_{a} w_{c}\left|\begin{array}{lll}
a & a & b \\
c & c & c
\end{array}\right|\left|\begin{array}{lll}
a & a & b \\
c & c & c
\end{array}\right|\left|\begin{array}{ccc}
b & b & b \\
a & a & a
\end{array}\right|\left(\sum_{d} w_{d} w_{b}\left|\begin{array}{lll}
b & b & b \\
a & a & d
\end{array}\right|\right),
\end{aligned}
$$



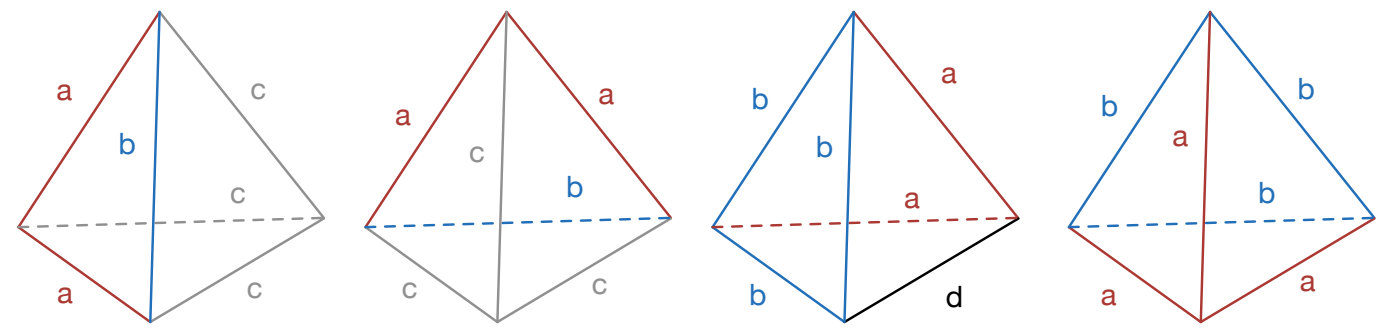

FIGURE 18.

where in the second row $a, b, c$ run over elements of $I_{r}$ such that all the involved triples are admissible. We claim that

$$
\sum_{d} w_{d} w_{b}\left|\begin{array}{lll}
b & b & b \\
a & a & d
\end{array}\right|=\sqrt{-1}^{2 a+2 b} \sqrt{[2 a+1][2 b+1]} \cdot \delta_{0, b} .
$$

Indeed, letting $m=0, i=j=a, k=l=n=b$ and $s=d$ in the Orthogonality Property (2.1), we have

$$
\sum_{d} w_{d} w_{b}\left|\begin{array}{lll}
b & b & b \\
a & a & 0
\end{array}\right|\left|\begin{array}{ccc}
b & b & b \\
a & a & d
\end{array}\right|=\delta_{0, b}
$$

Then the claim follows from the fact that

$$
\left|\begin{array}{lll}
b & b & b \\
a & a & 0
\end{array}\right|=\frac{\sqrt{-1}^{2 a+2 b}}{\sqrt{[2 a+1][2 b+1]}}
$$

Therefore,

$$
\begin{aligned}
\operatorname{TV}_{r}\left(T_{(2,4)}\right) & =\sum_{a, c} w_{a} w_{c}\left|\begin{array}{lll}
a & a & 0 \\
c & c & c
\end{array}\right|\left|\begin{array}{lll}
a & a & 0 \\
c & c & c
\end{array}\right|\left|\begin{array}{lll}
0 & 0 & 0 \\
a & a & a
\end{array}\right| \sqrt{-1}^{2 a} \sqrt{[2 a+1]} \\
& =\sum_{a, c}(-1)^{2 a}[2 a+1](-1)^{2 c}[2 c+1] \frac{(-1)^{2 a+2 c}}{[2 a+1][2 c+1]} \frac{\sqrt{-1}^{2 a}}{\sqrt{[2 a+1]}} \sqrt{-1}^{2 a} \sqrt{[2 a+1]} \\
& =\sum_{a, c} 1,
\end{aligned}
$$

where $a, c$ run over all the elements of $I_{r}$ such that $(c, c, a)$ and $(a, a, 0)$ are admissible. Counting the number of such pairs $(a, c)$, we have

$$
\mathrm{TV}_{r}\left(T_{(2,4)}\right)=\left(\left\lfloor\frac{r-2}{2}\right\rfloor+1\right)\left(\left\lfloor\frac{r-1}{2}\right\rfloor+1\right) .
$$

Proposition 5.8. Let $T_{(2,6)}$ be the $(2,6)$-torus link in $S^{3}$. Then

$$
\mathrm{TV}_{r}\left(S^{3} \backslash T_{(2,6)}\right)=\left(\left\lfloor\frac{r-2}{3}\right\rfloor+1\right)\left(\left\lfloor\frac{2 r-2}{3}\right\rfloor+1\right)
$$

for all $r \geqslant 3$ and for all $q \in \mathbb{C}$ such that $q^{2}$ is a primitive root of unity of degree $r$.

Proof. The complement of the torus link $T_{(2,6)}$ has the following ideal triangulation represented in Figure 19. 

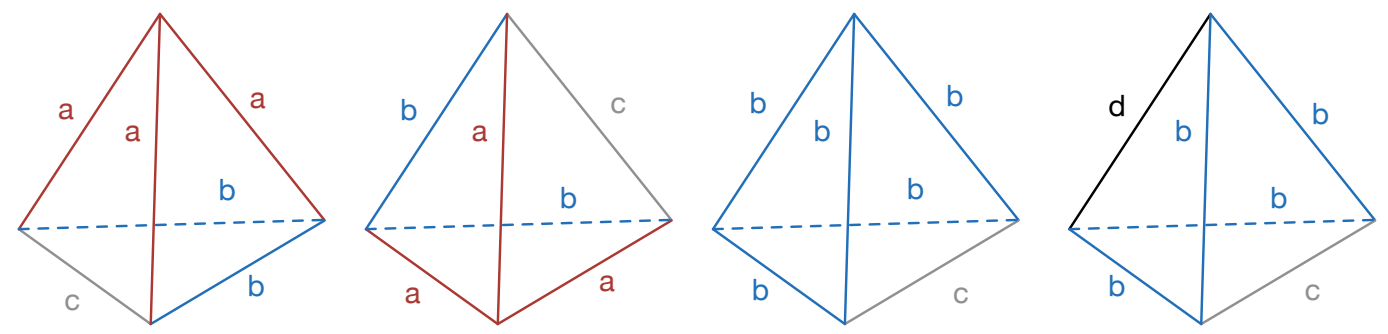

FIGURE 19.

Therefore, for each $r \geqslant 3$, we have

$$
\begin{aligned}
\operatorname{TV}_{r}\left(T_{(2,6)}\right) & =\sum_{(a, b, c, d) \in A_{r}} w_{a} w_{b} w_{c} w_{d}|| \begin{array}{lll}
a & a & c \\
b & b & a
\end{array}|| \begin{array}{lll}
a & a & c \\
b & b & a
\end{array}|| \begin{array}{lll}
b & b & c \\
b & b & b
\end{array}|| \begin{array}{lll}
b & b & d \\
b & b & c
\end{array} \mid \\
& =\sum_{a, b, c} w_{a} w_{b}\left|\begin{array}{lll}
a & a & c \\
b & b & a
\end{array}\right|\left|\begin{array}{lll}
a & a & c \\
b & b & a
\end{array}\right|\left|\begin{array}{lll}
b & b & c \\
b & b & b
\end{array}\right|\left(\sum_{d} w_{d} w_{c}\left|\begin{array}{lll}
b & b & d \\
b & b & c
\end{array}\right|\right),
\end{aligned}
$$

where in the second row $a, b, c$ run over elements of $I_{r}$ such that all the involved triples are admissible. We claim that

$$
\sum_{d} w_{d} w_{c}\left|\begin{array}{lll}
b & b & d \\
b & b & c
\end{array}\right|=(-1)^{2 b}[2 b+1] \cdot \delta_{0, c} .
$$

Indeed, letting $m=0, i=j=k=l=b, n=c$ and $s=d$ in the Orthogonality Property (2.1), we have

$$
\sum_{d} w_{d} w_{c}\left|\begin{array}{lll}
b & b & d \\
b & b & 0
\end{array}\right|\left|\begin{array}{lll}
b & b & d \\
b & b & c
\end{array}\right|=\delta_{0, c}
$$

Then the claim follows from the fact that

$$
\left|\begin{array}{lll}
b & b & d \\
b & b & 0
\end{array}\right|=\frac{(-1)^{2 b}}{[2 b+1]}
$$

Therefore,

$$
\begin{aligned}
\mathrm{TV}_{r}\left(T_{(2,6)}\right) & =\sum_{a, b} w_{a} w_{b}\left|\begin{array}{lll}
a & a & 0 \\
b & b & a
\end{array}\right|\left|\begin{array}{lll}
a & a & 0 \\
b & b & a
\end{array}\right|\left|\begin{array}{lll}
b & b & 0 \\
b & b & b
\end{array}\right|(-1)^{2 b}[2 b+1] \\
& =\sum_{a, b}(-1)^{2 a}[2 a+1](-1)^{2 b}[2 b+1] \frac{(-1)^{2 a+2 b}}{[2 a+1][2 b+1]} \frac{(-1)^{2 b}}{[2 b+1]}(-1)^{2 b}[2 b+1] \\
& =\sum_{a, b} 1
\end{aligned}
$$

where $a, b$ run over all the elements of $I_{r}$ such that $(a, a, b)$ and $(b, b, b)$ are admissible. Counting the number of such pairs $(a, b)$, we have

$$
\operatorname{TV}_{r}\left(T_{(2,6)}\right)=\left(\left\lfloor\frac{r-2}{3}\right\rfloor+1\right)\left(\left\lfloor\frac{2 r-2}{3}\right\rfloor+1\right) .
$$

Remark 5.9. Conjecture 1.1 can be generalized to non-hyperbolic 3-manifolds by considering the Gromov norm, and Propositions 5.2, 5.5, 5.6, 5.7, 5.8 prove that for the corresponding cases. 
VOLUME CONJECTURES FOR THE RESHETIKHIN-TURAEV AND THE TURAEV-VIRO INVARIANTS 25

5.2. Numerical evidence for Conjecture 5.1. In this subsection, we provide further evidence for Conjecture 5.1 by numerically calculating the Turaev-Viro invariants for the complements of the torus knots $T_{(2,5)}, T_{(3,5)}, T_{(2,7)}, T_{(3,7)}, T_{(2,9)}$ and $T_{(2,11)}$.

5.2.1. Knot $T_{(2,5)}$. The table below contains the values of $\operatorname{TV}_{r}\left(T_{(2,5)} ; e^{\frac{k \pi \sqrt{-1}}{r}}\right)$ for $k=1,2,3$ and $r \leqslant 20$.

\begin{tabular}{|c||c|c|c|c|c|c|c|c|c|c|c|c|c|c|c|c|c|c|c|}
\hline$k \backslash r$ & 3 & 4 & 5 & 6 & 7 & 8 & 9 & 10 & 11 & 12 & 13 & 14 & 15 & 16 & 17 & 18 & 19 & 20 \\
\hline 1 & 1 & 1 & 0.381966 & 1 & 3 & 3 & 2 & 0.763932 & 2 & 5 & 5 & 3 & 1.14590 & 3 & 7 & 7 & 4 & 1.52786 \\
\hline 2 & 1 & & 2.61803 & & 3 & & 2 & & 2 & & 5 & & 7.85410 & & 7 & \\
\hline 3 & & 1 & 2.61803 & & 3 & 3 & & 5.23607 & 2 & & 5 & 3 & & & 3 & 7 & & 4 & 10.4721 \\
\hline
\end{tabular}

5.2.2. Knot $T_{(3,5)}$. The table below contains the values of $\operatorname{TV}_{r}\left(T_{(3,5)} ; e^{\frac{k \pi \sqrt{-1}}{r}}\right)$ for $k=1,2,3$ and $r \leqslant 20$.

\begin{tabular}{|c||c|c|c|c|c|c|c|c|c|c|c|c|c|c|c|c|c|c|c|}
\hline$k \backslash r$ & 3 & 4 & 5 & 6 & 7 & 8 & 9 & 10 & 11 & 12 & 13 & 14 & 15 & 16 & 17 & 18 & 19 & 20 \\
\hline 1 & 1 & 1 & 0.381966 & 1 & 2 & 3 & 2 & 1.38197 & 2 & 4 & 4 & 3 & 1.76393 & 3 & 6 & 6 & 4 & 2.14590 \\
\hline 2 & 1 & & 2.61803 & & 2 & & 2 & & 2 & & 4 & & 6.23607 & & 6 & & 4 & \\
\hline 3 & & 1 & 2.61803 & & 2 & 3 & & 3.61803 & 2 & & 4 & 3 & & & 3 & 6 & & 4 & 8.85410 \\
\hline
\end{tabular}

5.2.3. Knot $T_{(2,7)}$. The table below contains the values of $\operatorname{TV}_{r}\left(T_{(2,7)} ; e^{\frac{k \pi \sqrt{-1}}{r}}\right)$ for $k=1,2,3$ and $r \leqslant 21$.

\begin{tabular}{|c||c|c|c|c|c|c|c|c|c|c|c|c|c|c|c|c|c|c|c|}
\hline$k \backslash r$ & 3 & 4 & 5 & 6 & 7 & 8 & 9 & 10 & 11 & 12 & 13 & 14 & 15 & 16 & 17 & 18 & 19 & 20 & 21 \\
\hline 1 & 1 & 1 & 2 & 1 & 0.307979 & 1 & 4 & 3 & 3 & 5 & 2 & 0.615957 & 2 & 7 & 5 & 5 & 8 & 3 & 0.923936 \\
\hline 2 & 1 & & 2 & & 0.643104 & & 4 & & 3 & & 2 & & 2 & & 5 & & 8 & \\
\hline 3 & & 1 & 2 & & 5.04892 & 1 & & 3 & 3 & & 2 & 10.0978 & & 7 & 5 & & 8 & 3 & \\
\hline
\end{tabular}

5.2.4. Knot $T_{(3,7)}$. The table below contains the values of $\operatorname{TV}_{r}\left(T_{(3,7)} ; e^{\frac{k \pi \sqrt{-1}}{r}}\right)$ for $k=1,2,3$ and $r \leqslant 21$.

\begin{tabular}{|c||c|c|c|c|c|c|c|c|c|c|c|c|c|c|c|c|c|c|c|}
\hline$k \backslash r$ & 3 & 4 & 5 & 6 & 7 & 8 & 9 & 10 & 11 & 12 & 13 & 14 & 15 & 16 & 17 & 18 & 19 & 20 & 21 \\
\hline 1 & 1 & 1 & 2 & 1 & 0.198062 & 1 & 3 & 3 & 3 & 4 & 2 & 0.841166 & 2 & 2 & 5 & 5 & 6 & 3 & 1.03923 \\
\hline 2 & 1 & & 2 & & 3.24698 & & 3 & & 3 & & 2 & & & & 5 & & 6 & & 11.5429 \\
\hline 3 & & 1 & 2 & & 1.55496 & 1 & & 3 & 3 & & 2 & 1.86294 & & 2 & 5 & & 6 & 3 & \\
\hline
\end{tabular}


5.2.5. Knot $T_{(2,9)}$. The table below contains the values of $\operatorname{TV}_{r}\left(T_{(2,9)} ; e^{\frac{k \pi \sqrt{-1}}{r}}\right)$ for $k=1,2,3$ and $r \leqslant 22$.

\begin{tabular}{|c|c|c|c|c|c|c|c|c|c|c|c|c|c|c|c|c|c|c|c|c|}
\hline$k \backslash r$ & 3 & 4 & 5 & 6 & 7 & 8 & 9 & 10 & 11 & 12 & 13 & 14 & 15 & 16 & 17 & 18 & 19 & 20 & 21 & 22 \\
\hline 1 & 1 & 1 & 1 & 2 & 3 & 1 & 0.283119 & 1 & 5 & 4 & 3 & 3 & 5 & 7 & 2 & 0.566237 & 2 & 9 & 7 & 5 \\
\hline 2 & 1 & & 1 & & 3 & & 0.426022 & & 5 & & 3 & & 5 & & 2 & & 2 & & 7 & \\
\hline 3 & & 1 & 1 & & 3 & 1 & & 1 & 5 & & 3 & 3 & & 7 & 2 & & 2 & 9 & & 5 \\
\hline
\end{tabular}

5.2.6. Knot $T_{(2,11)}$. The table below contains the values of $\operatorname{TV}_{r}\left(T_{(2,11)} ; e^{\frac{k \pi \sqrt{-1}}{r}}\right)$ for $k=1,2,3$ and $r \leqslant 22$.

\begin{tabular}{|c||c|c|c|c|c|c|c|c|c|c|c|c|c|c|c|c|c|c|c|c|c|}
\hline$k \backslash r$ & 3 & 4 & 5 & 6 & 7 & 8 & 9 & 10 & 11 & 12 & 13 & 14 & 15 & 16 & 17 & 18 & 19 & 20 & 21 & 22 \\
\hline 1 & 1 & 1 & 1 & 1 & 2 & 3 & 4 & 1 & 0.271554 & 1 & 6 & 5 & 4 & 3 & 3 & 5 & 7 & 9 & 2 & 0.543108 \\
\hline 2 & 1 & & 1 & & 2 & & 4 & & 0.353253 & & 6 & & 4 & & 3 & & 7 & & 2 & \\
\hline 3 & & 1 & 1 & & 2 & 3 & & 1 & 0.582964 & & 6 & 5 & & 3 & 3 & & 7 & 9 & & 1.16593 \\
\hline
\end{tabular}

\section{REFERENCES}

[1] J.E. Andersen and R. Kashaev, A TQFT from quantum Teichmüller theory, Comm. Math. Phys. 330(3):887-934, 2014.

[2] __ A new formulation of the Teichmüller TQFT, preprint: arXiv:1305.4291, 2013.

[3] _ Quantum Teichmüller theory and TQFT, XVIIth International Congress on Mathematical Physics, World Sci. Publ., Hackensack, NJ, 684-692, 2014.

[4] Complex quantum Chern-Simons, preprint: arXiv:1409.1208, 2014.

[5] _ Faddeev's quantum dilogarithm and state-integrals on shaped triangulations, In Mathematical Aspects of Quantum Field Theories, Editors D. Calaque and Thomas Strobl, Mathematical Physics Studies. XXVIII:133152, 2015.

[6] S. Baseilhac and R. Benedetti, Quantum hyperbolic invariants of 3-manifolds with PSL(2, C)-characters, Topology 43 (2004), no. 6, 1373-1423.

[7] _ Classical and quantum dilogarithmic invariants of flat PSL(2, C)-bundles over 3-manifolds, Geom. Topol. 9 (2005), 493-569.

[8] __ Quantum hyperbolic geometry, Algebr. Geom. Topol. 7 (2007), 845-917.

[9] _. The Kashaev and quantum hyperbolic link invariants, J. Gökova Geom. Topol. GGT 5 (2011), 31-85.

[10] R. Benedetti and C. Petronio, On Roberts' proof of the Turaev-Walker theorem, J. Knot Theory Ramifications 5 (1996), no. 4, 427-439.

[11] C. Blanchet, N. Habegger, G. Masbaum and P. Vogel, Three-manifold invariants derived from the Kauffman bracket, Topology 31 (1992), no. 4, 685-699.

[12] B. Burton, R. Budney and W. Pettersson, Regina, http://regina.sourceforge.net/.

[13] P.J. Callahan, J.C. Dean and J.R. Weeks, The simplest hyperbolic knots, J. Knot Theory Ramifications 8 (1999), no. 3, 279-297.

[14] P.J. Callahan, M.V. Hildebrand and J.R. Weeks, A census of cusped hyperbolic 3-manifolds, Math. Comp. 68 (1999), no. 225, 321-332.

[15] F. Costantino, Coloured Jones invariants of links and the volume conjecture, J. Lond. Math. Soc. (2) 76 (2007), no. $1,1-15$.

[16] _ 6j-symbols, hyperbolic structures and the volume conjecture, Geom. Topol. 11 (2007), 1831-1854.

[17] _ Integrality of Kauffman brackets of trivalent graphs, Quantum Topol. 5 (2014), no. 2, $143-184$.

[18] and J. Murakami, On the $S L(2, \mathbb{C})$ quantum $6 j$-symbols and their relation to the hyperbolic volume, Quantum Topol. 4 (2013), no. 3, 303-351.

[19] _ F. Guéritaud and R. van der Veen, On the volume conjecture for polyhedra, preprint: arXiv:1403.2347 
[20] _ N. Geer and B. Patureau-Mirand, Quantum invariants of 3-manifolds via link surgery presentations and non-semi-simple categories, J Topology (2014) 7 (4): 1005-1053.

[21] M. Culler and N. Dunfield, SnapPy, http:// sanppy.computop.org/.

[22] R. Detcherry, E. Kalfagianni, Efstratia and T. Yang, Turaev-Viro invariants, colored Jones polynomials and volume, preprint: arXiv:math/1701.07818.

[23] M. Fujii, Hyperbolic 3-manifolds with totally geodesic boundary which are decomposed into hyperbolic truncated tetrahedra, Tokyo J. Math. 13 (1990), no. 2, 353-373.

[24] S. Garoufalidis and T. Lê, On the volume conjecture for small angles, preprint: arXiv:math/0502163.

[25] _ Asymptotics of the colored Jones function of a knot, Geom. Topol. 15 (2011), no. 4, 2135-2180.

[26] S. Garoufalidis and R. van der Veen, Asymptotics of classical spin networks, with an appendix by D. Zagier. Geom. Topol. 17 (2013), no. 1, 1-37.

[27] __ Asymptotics of quantum spin networks at a fixed root of unity, Math. Ann. 352 (2012), no. 4, 987-1012.

[28] S. Gukov, Three-dimensional quantum gravity, Chern-Simons theory, and the A-polynomial, Comm. Math. Phys. 255 (2005) 577-627.

[29] R.M. Kashaev, A link invariant from quantum dilogarithm, Modern Phys. Lett. A 10 (1995), no. 19, 1409-1418.

[30] _ The hyperbolic volume of knots from the quantum dilogarithm, Lett. Math. Phys. 39 (1997), no. 3, 269-275.

[31] _ and O. Tirkkonen, A proof of the volume conjecture on torus knots, (Russian) Zap. Nauchn. Sem. S.Peterburg. Otdel. Mat. Inst. Steklov. (POMI) 269 (2000), Vopr. Kvant. Teor. Polya i Stat. Fiz. 16, 262-268, 370, translation in J. Math. Sci. (N. Y.) 115 (2003), no. 1, 2033-2036

[32] L. Kauffman and S. Lins, Temperley-Lieb recoupling theory and invariants of 3-manifolds, Annals of Mathematics Studies, 134. Princeton University Press, Princeton, NJ, 1994.

[33] R. Kirby, A calculus for framed links in $S^{3}$, Invent. Math. 45 (1978), 35-56.

[34] _ and P. Melvin, The 3-manifold invariants of Witten and Reshetikhin-Turaev for sl(2, C), Invent. Math. 105 (1991), no. 3, 473-545.

[35] A.A. Kirillov and N.Y. Reshetikhin, Representations of the algebra $U_{q}(s l(2))$, q-orthogonal polynomials and invariants of links, Infinite-dimensional Lie algebras and groups (Luminy-Marseille, 1988), 285-339, Adv. Ser. Math. Phys., 7, World Sci. Publ., Teaneck, NJ, 1989.

[36] W.B.R. Lickorish, Three-manifolds and the Temperley-Lieb algebra, Math. Ann. 290 (1991), no. 4, 657-670.

[37] __ Calculations with the Temperley-Lieb algebra. Comment. Math. Helv, 67 (1992), no. 4, 571-591.

[38] __ The skein method for three-manifold invariants, J. Knot Theory Ramifications 2 (1993), no. 2, 171-194.

[39] S. Kojima and Y. Miyamoto, The smallest hyperbolic 3-manifolds with totally geodesic boundary, J. Differential Geom. 34 (1991), no. 1, 175-192.

[40] G. Masbaum, Skein-theoretical derivation of some formulas of Habiro, Algebr. Geom. Topol. 3 (2003), 537-556.

[41] S.V. Matveev, Transformations of special spines, and the Zeeman conjecture, (Russian) Izv. Akad. Nauk SSSR Ser. Mat. 51 (1987), no. 5, 1104-1116, 1119, translation in Math. USSR-Izv. 31 (1988), no. 2, 423-434.

[42] __ Algorithmic topology and classification of 3-manifolds, second ed., Algorithms and Com- putation in Mathematics, vol. 9, Springer, Berlin, 2007.

[43] H. Murakami, An introduction to the volume conjecture, Interactions between hyperbolic geometry, quantum topology and number theory, 1-40, Contemp. Math., 541, Amer. Math. Soc., Providence, RI, 2011.

[44] __ and J. Murakami, The colored Jones polynomials and the simplicial volume of a knot, Acta Math. 186 (2001), no. 1, 85-104.

[45] , M. Okamoto, T. Takata and Y. Yokota, Kashaev's conjecture and the Chern-Simons invariants of knots and links, Experiment. Math. 11 (2002), no. 3, 427-435.

[46]__ and Y. Yokota, The colored Jones polynomials of the figure-eight knot and the volumes of three-manifolds obtained by Dehn surgeries, J. Reine Angew. Math. 607 (2007), 47-68.

[47] J. Murakami, Generalized Kashaev invariants for knots in three manifolds, preprint: arXiv:1312.0330

[48] T. Ohtsuki, Problems on invariants of knots and 3-manifolds, with an introduction by J. Roberts. Geom. Topol. Monogr., 4, Invariants of knots and 3-manifolds (Kyoto, 2001), i-iv, 377-572, Geom. Topol. Publ., Coventry, 2002.

[49] _ On the asymptotic expansion of the Kashaev invariant of the $5_{2}$ knot, to appear in Quantum Topology.

[50] _ and Y. Yokota, On the asymptotic expansions of the Kashaev invariant of the knots with 6 crossings, preprint.

[51] , private communication. 
[52] R. Piergallini, Standard moves for standard polyhedra and spines, Third National Conference on Topology (Italian) (Trieste, 1986). Rend. Circ. Mat. Palermo (2) Suppl. No. 18 (1988), 391-414.

[53] N.Y. Reshetikhin and V.G. Turaev, Ribbon graphs and their invariants derived from quantum groups, Comm. Math. Phys. 127 (1990), no. 1, 1-26.

[54] _ Invariants of 3-manifolds via link polynomials and quantum groups, Invent. Math. 103 (1991), no. 3, $547-597$.

[55] J. Roberts, Skein theory and Turaev-Viro invariants, Topology 34 (1995), no. 4, 771-787.

[56] W.P. Thurston, Geometry and topology of 3-manifolds, Revised Lecture Notes, Princeton Univ. Math. Dept. (1982)

[57] V.G. Turaev, Shadow links and face models of statistical mechanics, J. Differential Geom. 36 (1992), no. 1, $35-74$.

[58] _ Quantum invariants of links and 3-valent graphs in 3-manifolds, Inst. Hautes tudes Sci. Publ. Math. No. 77 (1993), 121-171.

[59] __ Quantum invariants of knots and 3-manifolds, Second revised edition. de Gruyter Studies in Mathematics, 18. Walter de Gruyter \& Co., Berlin, 2010.

[60] and O.Y. Viro, State sum invariants of 3-manifolds and quantum $6 j$-symbols, Topology 31 (1992), no. 4, $865-902$.

[61] K. Walker, On Wittens 3-manifold invariants, preprint.

[62] E. Witten, Quantum Field Theory and the Jones Polynomial, Comm. Math. Phys. 121 (3): 351-399.

[63] R. van der Veen, Proof of the volume conjecture for Whitehead chains, Acta Math. Vietnam. 33 (2008), no. 3, $421-431$.

[64] _ The volume conjecture for augmented knotted trivalent graphs, Algebr. Geom. Topol. 9 (2009), no. 2 , 691-722.

[65] D. Zagier, Quantum modular forms, Quanta of Maths: Conference in honor of Alain Connes, Clay Mathematics Proceedings 11, AMS and Clay Mathematics Institute 2010, 659-675.

[66] H. Zheng, Proof of the volume conjecture for Whitehead doubles of a family of torus knots, Chin. Ann. Math. Ser. B 28 (2007), no. 4, 375-388.

Department of Mathematics, ETH Zurich, 8092 Zurich, SwitZerland

E-mail address: qingtao.chen@math.ethz.ch

Department of Mathematics, Stanford University, Stanford CA 94305, USA

E-mail address: yangtian@math.stanford.edu 